\title{
Chemical equilibrium study in nucleus-nucleus collisions at relativistic energies
}

\author{
F. Becattini \\ Università di Firenze and INFN Sezione di Firenze, Florence, Italy \\ M. Gaździcki \\ Institut für Kernphysik, Universität Frankfurt, Frankfurt, Germany and Świętokrzyska Academy, Kielce, Poland \\ A. Keränen \\ University of Oulu, Oulu, Finland and INFN Sezione di Firenze, Florence, Italy \\ J. Manninen \\ University of Oulu, Oulu, Finland \\ R. Stock \\ Institut für Kernphysik, Universität Frankfurt, Frankfurt, Germany and CERN, Geneva, Switzerland
}

(Received 9 October 2003; published 26 February 2004)

\begin{abstract}
We present a detailed study of chemical freeze-out in nucleus-nucleus collisions at beam energies of $11.6 \mathrm{~A}$, $30 A, 40 A, 80 A$, and $158 \mathrm{~A} \mathrm{GeV}$. By analyzing hadronic multiplicities within the statistical hadronization approach, we have studied the strangeness production as a function of center-of-mass energy and of the parameters of the source. We have tested and compared different versions of the statistical model, with special emphasis on possible explanations of the observed strangeness hadronic phase space undersaturation. We show that, in this energy range, the use of hadron yields at midrapidity instead of in full phase space artificially enhances strangeness production and could lead to incorrect conclusions as far as the occurrence of full chemical equilibrium is concerned. In addition to the basic model with an extra strange quark nonequilibrium parameter, we have tested three more schemes: a two-component model superimposing hadrons coming out of single nucleon-nucleon interactions to those emerging from large fireballs at equilibrium, a model with local strangeness neutrality and a model with strange and light quark nonequilibrium parameters. The behavior of the source parameters as a function of colliding system and collision energy is studied. The description of strangeness production entails a nonmonotonic energy dependence of strangeness saturation parameter $\gamma_{S}$ with a maximum around $30 \mathrm{~A} \mathrm{GeV}$. We also present predictions of the production rates of still unmeasured hadrons including the newly discovered $\Theta^{+}(1540)$ pentaquark baryon.
\end{abstract}

DOI: 10.1103/PhysRevC.69.024905

PACS number(s): 25.75.Dw, 25.75.Nq, 12.40.Ee

\section{INTRODUCTION}

The main goal of the ultrarelativistic nucleus-nucleus (A-A) collisions programme is to create in terrestrial laboratories a new state of matter, the quark-gluon plasma (QGP). The existence of this phase, where quarks and gluons are deconfined, i.e., can freely move over several hadronic distances, is a definite prediction of quantum chromodynamics. In a search for QGP signals A-A collisions at different center-of-mass energies per nucleon-nucleon $(\mathrm{NN})$ pair have been studied: from few $\mathrm{GeV}$ to several hundreds of $\mathrm{GeV}$ recently attained in Au-Au collisions at RHIC.

Recently, accurate measurements of hadron production in central $\mathrm{Pb}-\mathrm{Pb}$ collisions at $40 \mathrm{~A}, 80 \mathrm{~A}$, and $158 \mathrm{~A} \mathrm{GeV}$ of beam energy became available [1] and also preliminary data at $30 \mathrm{~A} \mathrm{GeV}$ have been presented [2] following an energy scan programme carried out by the experiment NA49 at CERN SPS. This programme is motivated by the hypothesis [3] that the threshold for creation of QGP in the early stage of $\mathrm{Pb}-\mathrm{Pb}$ collisions might be located in the low SPS energy range, roughly between $20 \mathrm{~A}$ and $40 \mathrm{~A} \mathrm{GeV}$ of beam energy.

One of the main results of the study of high energy A-A collisions is a surprising success of the statistical-thermal models in reproducing essential features of particle production [4-11]. This model succeeds also in describing particle multiplicities in many kinds of elementary collisions [12-14], suggesting that statistical production is a general property of the hadronization process itself $[13,15]$. Furthermore, the statistical hadronization model (SHM) supplemented with the hydrodynamical expansion of the matter, to a large extent, also reproduces transverse momentum spectra of different particle species [16].

Hence, the SHM model proves to be a useful tool for the analysis of soft hadron production and particularly to study strangeness production, whose enhancement has since long been proposed as a signature of QGP formation. Furthermore, anomalies in the energy dependence of strangeness production have been predicted as a signature of deconfinement and have been indeed observed experimentally [17], suggesting that the onset of the phase transition could be located around $30 \mathrm{~A} \mathrm{GeV}$. It is thus important to make a systematic analysis, within the framework of SHM, of the presently available hadronic multiplicities measured in $\mathrm{Pb}-\mathrm{Pb}$ collisions at $30 \mathrm{~A}, 40 \mathrm{~A}$, and $80 \mathrm{~A} \mathrm{GeV}$, which-to our knowledge-is done here for the first time.

Along with these intriguing questions, our work is also 
motivated by issues related to the application of statistical model itself. In fact, different versions of this model have been used in the past by different authors leading to somewhat different results and conclusions. These mainly stem from the alternative use of midrapidity and full phase space multiplicities, from the allowance of nonequilibrium abundances of hadrons, from the assumption of exact local vanishing strangeness, etc. Therefore, we consider the comparison of these different approaches a worthwhile step. This has been made possible by now by the availability of an accurate and large multiplicity sample in $\mathrm{Pb}-\mathrm{Pb}$ collisions at $158 \mathrm{~A} \mathrm{GeV}$ as well as the corresponding data for $p p$ interactions.

The paper is organized as follows. A brief description of our main version of the SHM is given in Sec. II. The experimental data selected for the analysis are summarized in Sec. III. In Sec. IV the results of the analysis using the main version and alternative schemes of the SHM are given. Finally, in Sec. V we present and discuss the energy dependence of the chemical freeze-out stage. Summary and conclusions are drawn in Sec. VI.

\section{THE STATISTICAL HADRONIZATION MODEL}

The main idea of the SHM is that hadrons are emitted from regions at statistical equilibrium, called clusters or fireballs. No hypothesis is made about how statistical equilibrium is achieved; this can be a direct consequence of the hadronization process. In a single collision event, there might be several clusters with different collective momenta, different overall charges and volumes. However, Lorentzinvariant quantities such as particle multiplicities are independent of cluster momenta, while they depend on charges and volumes. If final state interactions among formed hadrons occur, particle multiplicities are frozen when inelastic interactions cease (chemical freeze-out). Thus, by analyzing measured hadron abundances, a snapshot is taken of clusters at that particular stage of the evolution, which may significantly precede the final kinetic freeze-out stage, when also elastic interactions cease. However, it should be pointed out that chemical and kinetic freeze-out may depend on the hadron species and the assumption of a single chemical freezeout is certainly an approximation. Most calculations in SHM are carried out in the framework of the ideal hadronresonance gas, that is, handling resonances as free particles: this amounts to taking a considerable part of the hadronic interactions between strongly stable hadrons into account [18].

As has been mentioned, final multiplicities depend on the distribution of initial conserved charges (baryon number, strangeness, and electric charge) among the produced clusters. This distribution is determined by the dynamics of the collision and is thus needed as an external input to the statistical model. However, most analyses, including ours, are carried out by assuming a single fireball. This is possible provided that one of the two conditions below is fulfilled.

(1) All clusters are large enough to allow a grandcanonical description and all of them have the same values of relevant intensive parameters, i.e., temperature and chemical potentials.
(2) Clusters are small and must be treated canonically (i.e., counting those states having exactly the same charges as the cluster itself), yet they have the same temperature and the distribution governing fluctuations of charges is the same as that obtained by splitting one large cluster-the equivalent global cluster (EGC) - having as volume the sum of all cluster rest frame volumes and as charges the sum of all cluster charges (see Appendix A). In this case the overall particle multiplicities turn out to be those calculated in the canonical, perhaps grand-canonical, ensemble of the equivalent global cluster [13]. The reduction to EGC could be achieved even for microcanonical clusters with additional requirement on mass fluctuations [14].

The first condition sets stronger requirements and applies in Bjorken's boost-invariant scenario, where all clusters are to have the same parameters independently of their rapidity. The second condition is altogether weaker and leaves room for the compatibility between the single fireball analysis and a variation of net baryon number density in rapidity. This has been discussed in detail in Ref. [6]. The argument can be summarized as follows: particle multiplicities, being Lorentz invariants, are unaffected by a shift in rapidity of the clusters, therefore, clusters arising from the splitting of the EGC can be ordered in rapidity according to their net baryon number without affecting fully integrated particle multiplicities and, at the same time, giving rise to an effective variation of the baryon density profile. Although the second condition is certainly more appropriate in the examined energy range, from AGS to SPS, it must be pointed out that this should not be expected to precisely match physical reality, as well as the first condition in its domain of applicability. In other words, discrepancies (hopefully small) between calculations based on this model and measurement are to be expected, so that these analyses shall not provide perfect fits even though the statistical model was the underlying true model.

In this paper we will stick to the picture outlined in the second condition, which implicitly requires the use of full phase space multiplicities in order to (hopefully) integrate out correlations between clusters' momenta and charges. Besides their general fitness, $4 \pi$ multiplicities also allow us to safely enforce overall strangeness neutrality. As has been mentioned, if the second condition applies, the multiplicity of any hadron $j$ can be calculated in the canonical ensemble of the EGC. Hence, as the EGC has a much larger volume than single clusters', the grand-canonical ensemble, where charges are conserved on average, can be a good approximation (see Appendix A). This is the case for the collisions examined in this paper [19]. In this case the mean primary multiplicity of the $j$ th hadron with mass $m_{j}$ and $\operatorname{spin} J_{j}$ reads

$$
\left\langle n_{j}\right\rangle=\frac{\left(2 J_{j}+1\right) V}{(2 \pi)^{3}} \int d^{3} p\left[e^{\sqrt{p^{2}+m_{j}^{2}} / T-\mu \cdot \mathbf{q}_{j} / T} \pm 1\right]^{-1},
$$

where $T$ is the temperature, $V$ the EGC volume, $\mathbf{q}_{j}$ $=\left(Q_{j}, B_{j}, S_{j}\right)$ is a vector having as components the electric charge, baryon number, and strangeness of the hadron, and $\boldsymbol{\mu}=\left(\mu_{Q}, \mu_{B}, \mu_{S}\right)$ is a vector of the corresponding chemical potentials; the upper sign applies to fermions, the lower to bosons. In order to correctly reproduce the data, it is also 
necessary to introduce at least one nonequilibrium parameter suppressing hadrons containing valence strange quarks, $\gamma_{S}$ $\neq 1$ [20]. With this supplementary parameter, hadron multiplicity is as in Eq. (1) with the replacement

$$
\exp \left[\boldsymbol{\mu} \cdot \mathbf{q}_{j} / T\right] \rightarrow \exp \left[\boldsymbol{\mu} \cdot \mathbf{q}_{j} / T\right] \gamma_{S}^{n_{s}},
$$

where $n_{s}$ stands for the number of valence strange quarks and antiquarks in the hadron $j$.

The abundances of resonances is calculated convoluting Eq. (1) with a relativistic Breit-Wigner distribution over a mass interval $[m-\delta m, m+\delta m]$, where $\delta m=\min [m$ $\left.-m_{\text {threshold }}, 2 \Gamma\right]$. The minimum mass $m_{\text {threshold }}$ is needed to open all decay modes. Finally, the overall multiplicity to be compared with the data is calculated as the sum of primary multiplicity (1) and the contribution from the decay of heavier hadrons:

$$
\left\langle n_{j}\right\rangle=\left\langle n_{i}\right\rangle^{\text {primary }}+\sum_{k} \operatorname{Br}(k \rightarrow j)\left\langle n_{k}\right\rangle,
$$

where the branching ratios (Br's) are taken from the latest issue of the Review of Particle Physics [21] and the summation runs over decays which contribute to the experimentally measured multiplicity. Among the hadrons and resonances contributing to the sum in Eq. (3), in this work all known states quoted in Ref. [21] up to a mass of $1.8 \mathrm{GeV}$ are included (see discussion in Sec. IV).

What we have hitherto described is the main version of the SHM used for the data analysis, which will be henceforth referred to as $\operatorname{SHM}\left(\gamma_{S}\right)$. As has been mentioned in the Introduction, in this work we also test other schemes and versions of the SHM, which will be described in detail in Sec. IV.

\section{EXPERIMENTAL DATA SET}

The bulk of the experimental data consists of measurements made by NA49 collaboration in central $\mathrm{Pb}-\mathrm{Pb}$ collisions at beam momenta of $30 \mathrm{~A}, 40 \mathrm{~A}, 80 \mathrm{~A}$, and $158 \mathrm{~A} \mathrm{GeV}$, corresponding to $\sqrt{s}_{N N}=7.6,8.8,12.3$, and $17.2 \mathrm{GeV}$, respectively. The acceptance region in rapidity and transverse momentum covers a typical range from midrapidity to projectile rapidity and from 0 to $1.5 \mathrm{GeV} / c$, respectively. The overall hadron multiplicities, quoted in referenced papers, were obtained using forward-backward symmetry in rapidity and by extrapolating the yields to full phase space. All results were corrected for the feed-down from weak decays, e.g., $\pi^{-}$multiplicity does not include pions produced in decays of $\Lambda$ hyperons and $K_{S}^{0}$ mesons.

Central collisions were selected by a trigger using information from a downstream calorimeter, which measured the energy of the projectile spectator nucleons. While at $30 \mathrm{~A}$, $40 \mathrm{~A}$, and $80 \mathrm{~A} \mathrm{GeV}$ all published results refer to the $7.2 \%$ most central collision sample, at $158 \mathrm{~A} \mathrm{GeV}$ different centrality selections $(5 \%, 10 \%$, and $20 \%$ most central collisions) were used to measure various hadronic species. In this analysis we have rescaled all published multiplicities at $158 \mathrm{~A} \mathrm{GeV}$ to the corresponding ones at $5 \%$ most central collisions assuming that for the considered central collisions the hadron yield is proportional to the mean number of par- ticipant nucleona. The resulting scaling factors are 1.08 and 1.32 for $10 \%$ and $20 \%$ most central collisions, respectively $[1,22]$.

As far as AGS data at $11.6 \mathrm{~A} \mathrm{GeV}$ is concerned, we have used both multiplicities measured by the experiments and extrapolations of measured rapidity distributions made in Ref. [9] at 3\% top centrality. For $\Lambda$ we have made a weighted average of the multiplicities measured both at 5\% top centrality by E896 [23] and E891 [24]. For the former, the quoted experimental error was only statistical so that we have added a $10 \%$ systematic error resulting in a value of $16.7 \pm 0.5 \pm 1.7$. For the latter, we have used the extrapolated value in Ref. [9] of $20.34 \pm 2.74$. The error on the weighted average has been rescaled by 1.25 (i.e., $\sqrt{\chi^{2}}$ ) according to the PDG weighting method in case of discrepancy between different measurements [21]. The obtained average has been rescaled by a factor of 1.02 to convert it from 5\% to $3 \%$ top centrality by assuming a linear dependence on the number of participants and by using the tables in Ref. [25]. Since the $\bar{\Lambda}$ to $\Lambda$ ratio has been measured only at midrapidity [26], we have obtained a $\bar{\Lambda} 4 \pi$ multiplicity assuming that the double ratio $(\langle\bar{\Lambda}\rangle /\langle\Lambda\rangle)_{y=0} /(\langle\bar{\Lambda}\rangle /\langle\Lambda\rangle)$ is the same at SPS and AGS energies. The final experimental multiplicities and ratios used in our analysis are shown in Tables I-V.

In order to test the effect of the cut in rapidity on the resulting statistical model parameters (discussed in detail in Sec. IV) we have also determined the yields integrated over limited $(\Delta y=1$ and $\Delta y=2)$ rapidity windows around midrapidity in $\mathrm{Pb}-\mathrm{Pb}$ collisions at $158 \mathrm{~A} \mathrm{GeV}$. This has been done by fitting the rapidity distributions measured by NA49 to a Gaussian or the sum of two Gaussians, with area and width as free parameters and central values set to zero. The results are shown in Table VI. The quality of the fits is quite good, except for pions due to a couple of points near midrapidity; yet, this discrepancy does not affect significantly the integrated yield. In fact, it must be stressed that the main goal of these fits is to estimate an integral and not to reproduce accurately the shape of the distributions over the full measured range. We have also checked that the extrapolations to full phase space are in good agreement with published measurements.

\section{ANALYSIS RESULTS}

The analysis has been carried out by looking for the minima of $\chi^{2}$,

$$
\chi^{2}=\sum_{i} \frac{\left(n_{i}^{\text {exp }}-n_{i}^{\text {theo }}\right)^{2}}{\sigma_{i}^{2}},
$$

where $n_{i}$ is the multiplicity of the $i$ th measured hadronic species and $\sigma_{i}$ is the sum in quadrature of statistical and systematic experimental error.

The theoretical multiplicities are calculated according to Eq. (3) with the decay chain stopped to match the experimental definition of multiplicity to properly compare theoretical and experimental values. This occurs in $\mathrm{Pb}-\mathrm{Pb}$ collisions after electromagnetic and strong decays and before weak de- 
TABLE I. Comparison between measured and fitted particle multiplicities, in the framework of $\operatorname{SHM}\left(\gamma_{S}\right)$ model in central $\mathrm{Au}-\mathrm{Au}$ collisions $(3 \%)$ at a beam energy of $11.6 \mathrm{~A} \mathrm{GeV}$. Also shown are the predicted multiplicities of the main hadron species.

\begin{tabular}{|c|c|c|c|c|}
\hline & Reference & Measurement & Fit A & Fit B \\
\hline$N_{P}$ & [39] & $363 \pm 10$ & 361.7 & 360.6 \\
\hline$p / \pi^{+}$ & {$[9,25]$} & $1.23 \pm 0.13$ & 1.277 & 1.224 \\
\hline$\pi^{+}$ & {$[9,40]$} & $133.7 \pm 9.93$ & 134.9 & 140.0 \\
\hline$\pi^{-}$ & & & 176.9 & 182.2 \\
\hline$\pi^{0}$ & & & 163.5 & 163.2 \\
\hline$K^{+}$ & [39] & $23.7 \pm 2.86$ & 18.80 & 18.81 \\
\hline$K^{-}$ & [39] & $3.76 \pm 0.47$ & 3.890 & 3.539 \\
\hline$K_{S}^{0}$ & & & 11.68 & 11.68 \\
\hline$\eta$ & & & 8.073 & 6.340 \\
\hline$\omega$ & & & 4.870 & 3.659 \\
\hline$\phi$ & & & 0.3287 & 0.3489 \\
\hline$\eta^{\prime}$ & & & 0.2997 & 0.2437 \\
\hline$\rho^{+}$ & & & 7.707 & 10.39 \\
\hline$\rho^{-}$ & & & 9.164 & 12.55 \\
\hline$\rho^{0}$ & & & 8.517 & 11.59 \\
\hline$K^{*+}$ & & & 3.555 & 3.512 \\
\hline$K^{*-}$ & & & 0.6179 & 0.5145 \\
\hline$K^{* 0}$ & & & 3.766 & 3.801 \\
\hline $\bar{K}^{* 0}$ & & & 0.5555 & 0.4628 \\
\hline$p$ & & & 172.2 & 171.4 \\
\hline $\bar{p}$ & & & 0.02851 & 0.02465 \\
\hline$\Delta^{++}$ & & & 25.39 & 24.51 \\
\hline $\bar{\Delta}^{--}$ & & & 0.003071 & 0.00222 \\
\hline$\Lambda$ & {$[23,24]$, see text } & $18.1 \pm 1.9$ & 19.82 & 20.71 \\
\hline $\bar{\Lambda}$ & & $0.017 \pm 0.005$ & 0.01601 & 0.01645 \\
\hline$\Sigma^{+}$ & & & 4.840 & 4.784 \\
\hline$\Sigma^{-}$ & & & 5.457 & 5.453 \\
\hline$\Sigma 0$ & & & 5.163 & 5.106 \\
\hline $\bar{\Sigma}^{-}$ & & & 0.003445 & 0.00321 \\
\hline $\bar{\Sigma}^{+}$ & & & 0.002793 & 0.00259 \\
\hline $\bar{\Sigma}^{0}$ & & & 0.003115 & 0.00288 \\
\hline$\Xi^{-}$ & & & 0.56067 & 0.5564 \\
\hline$\Xi^{0}$ & & & 0.54670 & 0.5387 \\
\hline $\bar{\Xi}^{+}$ & & & 0.002133 & 0.00248 \\
\hline $\bar{\Xi}^{0}$ & & & 0.002392 & 0.00280 \\
\hline$\Omega$ & & & 0.01352 & 0.01459 \\
\hline $\bar{\Omega}$ & & & 0.0003569 & 0.00056 \\
\hline$\Lambda(1520)$ & & & 0.7720 & 0.6601 \\
\hline$\Theta^{+}(1540)$ & & & 1.86 & 2.20 \\
\hline $\bar{\Theta}^{-}(1540)$ & & & $2.710^{-5}$ & $1.8710^{-5}$ \\
\hline
\end{tabular}

cays, while in Au-Au collisions at AGS the weak decays of $\Lambda, \Sigma, \Xi, \Omega$, and $K_{S}^{0}$ are included.

The effect of the uncertainties on masses, widths, and branching ratios of the involved hadrons on the fit param-
TABLE II. Comparison between measured and fitted particle multiplicities, in the framework of $\operatorname{SHM}\left(\gamma_{S}\right)$ model, in central $\mathrm{Pb}-\mathrm{Pb}$ collisions $(7.2 \%)$ at a beam energy of $30 \mathrm{~A} \mathrm{GeV}$. Also shown are the predicted multiplicities of the main hadron species.

\begin{tabular}{|c|c|c|c|c|}
\hline & Reference & Measurement & Fit A & Fit B \\
\hline$N_{P}$ & {$[2]$} & $349 \pm 1 \pm 5$ & 350.5 & 350.5 \\
\hline$\pi^{+}$ & [2] & $239 \pm 0.7 \pm 17$ & 228.4 & 228.5 \\
\hline$\pi^{-}$ & {$[2]$} & $275 \pm 0.7 \pm 20$ & 256.5 & 256.8 \\
\hline$\pi^{0}$ & & & 265.8 & 251.9 \\
\hline$K^{+}$ & {$[2]$} & $55.3 \pm 1.6 \pm 2.8$ & 49.83 & 48.83 \\
\hline$K^{-}$ & {$[2]$} & $16.1 \pm 0.2 \pm 0.8$ & 17.11 & 20.72 \\
\hline$K_{S}^{0}$ & & & 33.57 & 34.85 \\
\hline$\eta$ & & & 23.74 & 21.60 \\
\hline$\omega$ & & & 15.45 & 12.99 \\
\hline$\phi$ & & & 2.571 & 2.848 \\
\hline$\eta^{\prime}$ & & & 1.411 & 1.341 \\
\hline$\rho^{+}$ & & & 20.24 & 22.68 \\
\hline$\rho^{-}$ & & & 23.09 & 26.05 \\
\hline$\rho^{0}$ & & & 22.14 & 25.11 \\
\hline$K^{*+}$ & & & 13.65 & 13.45 \\
\hline$K^{*-}$ & & & 4.006 & 3.668 \\
\hline$K^{* 0}$ & & & 14.21 & 14.20 \\
\hline $\bar{K}^{* 0}$ & & & 3.710 & 3.386 \\
\hline$p$ & & & 138.0 & 137.0 \\
\hline $\bar{p}$ & & & 0.3650 & 0.3803 \\
\hline$\Delta^{++}$ & & & 26.90 & 25.23 \\
\hline $\bar{\Delta}^{--}$ & & & 0.07781 & 0.07438 \\
\hline$\Lambda$ & & & 38.02 & 40.25 \\
\hline $\bar{\Lambda}$ & & & 0.3393 & 0.3901 \\
\hline$\Sigma^{+}$ & & & 9.995 & 9.935 \\
\hline$\Sigma^{-}$ & & & 10.91 & 10.89 \\
\hline$\Sigma^{0}$ & & & 10.48 & 10.40 \\
\hline $\bar{\Sigma}^{-}$ & & & 0.1005 & 0.1106 \\
\hline $\bar{\Sigma}^{+}$ & & & 0.08620 & 0.09491 \\
\hline $\bar{\Sigma}^{0}$ & & & 0.09334 & 0.1023 \\
\hline$\Xi^{-}$ & & & 2.422 & 2.422 \\
\hline$\Xi^{0}$ & & & 2.378 & 2.369 \\
\hline $\bar{\Xi}^{+}$ & & & 0.07920 & 0.09332 \\
\hline $\bar{\Xi}^{0}$ & & & 0.08446 & 0.09967 \\
\hline$\Omega$ & & & 0.1587 & 0.1799 \\
\hline $\bar{\Omega}$ & & & 0.02067 & 0.02959 \\
\hline$\Lambda(1520)$ & & & 2.167 & 1.751 \\
\hline$\Theta^{+}(1540)$ & & & 2.84 & 3.02 \\
\hline $\bar{\Theta}^{-}(1540)$ & & & 0.0018 & 0.0019 \\
\hline
\end{tabular}

eters has been studied by the method described in Ref. [14] and found to be negligible throughout.

In order to cross-check our results and verify their robustness, we have performed the analysis with two independent numerical programs, henceforth referred to as $\mathrm{A}$ and $\mathrm{B}$, 
TABLE III. Comparison between measured and fitted particle multiplicities, in the framework of $\operatorname{SHM}\left(\gamma_{S}\right)$ model, in central $\mathrm{Pb}-\mathrm{Pb}$ collisions $(7.2 \%)$ at a beam energy of $40 \mathrm{~A} \mathrm{GeV}$. Also shown are the predicted multiplicities of the main hadron species.

\begin{tabular}{|c|c|c|c|c|}
\hline & Reference & Measurement & Fit A & Fit B \\
\hline$N_{P}$ & [1] & $349 \pm 1 \pm 5$ & 352.1 & 351.6 \\
\hline$\pi^{+}$ & [1] & $293 \pm 3 \pm 15$ & 285.5 & 288.3 \\
\hline$\pi^{-}$ & [1] & $322 \pm 3 \pm 16$ & 314.7 & 317.9 \\
\hline$\pi^{0}$ & & & 330.4 & 314.9 \\
\hline$K^{+}$ & [1] & $59.1 \pm 1.9 \pm 3$ & 51.22 & 50.61 \\
\hline$K^{-}$ & [1] & $19.2 \pm 0.5 \pm 1.0$ & 20.52 & 20.33 \\
\hline$K_{S}^{0}$ & & & 35.79 & 36.24 \\
\hline$\eta$ & & & 30.48 & 26.59 \\
\hline$\omega$ & & & 23.00 & 19.57 \\
\hline$\phi$ & {$[22]$} & $2.57 \pm 0.10$ & 2.641 & 2.644 \\
\hline$\eta^{\prime}$ & & & 1.858 & 1.621 \\
\hline$\rho^{+}$ & & & 28.84 & 31.11 \\
\hline$\rho^{-}$ & & & 32.36 & 35.01 \\
\hline$\rho^{0}$ & & & 31.27 & 33.97 \\
\hline$K^{*+}$ & & & 15.55 & 14.34 \\
\hline$K^{*-}$ & & & 5.393 & 4.860 \\
\hline$K^{* 0}$ & & & 16.06 & 14.98 \\
\hline $\bar{K}^{* 0}$ & & & 5.045 & 4.535 \\
\hline$p$ & & & 141.7 & 141.9 \\
\hline $\bar{p}$ & & & 0.9824 & 0.9784 \\
\hline$\Delta^{++}$ & & & 29.05 & 27.03 \\
\hline $\bar{\Delta}^{--}$ & & & 0.2181 & 0.1969 \\
\hline$\Lambda$ & {$[28]$} & $45.6 \pm 3.4$ & 36.60 & 37.36 \\
\hline $\bar{\Lambda}$ & [28] & $0.74 \pm 0.06$ & 0.7223 & 0.7297 \\
\hline$\Sigma^{+}$ & & & 9.655 & 9.221 \\
\hline$\Sigma^{-}$ & & & 10.40 & 9.938 \\
\hline$\Sigma^{0}$ & & & 10.05 & 9.567 \\
\hline $\bar{\Sigma}^{-}$ & & & 0.2116 & 0.2033 \\
\hline $\bar{\Sigma}^{+}$ & & & 0.1853 & 0.1783 \\
\hline $\bar{\Sigma}^{0}$ & & & 0.1985 & 0.1901 \\
\hline$\Xi^{-}$ & & & 2.118 & 1.948 \\
\hline$\Xi^{0}$ & & & 2.089 & 1.917 \\
\hline $\bar{\Xi}^{+}$ & & & 0.1285 & 0.1207 \\
\hline $\bar{\Xi}^{0}$ & & & 0.1358 & 0.1277 \\
\hline$\Omega$ & & & 0.1364 & 0.1344 \\
\hline $\bar{\Omega}$ & & & 0.02719 & 0.02788 \\
\hline$\Lambda(1520)$ & & & 2.273 & 1.688 \\
\hline$\Theta^{+}(1540)$ & & & 2.61 & 2.32 \\
\hline $\bar{\Theta}^{-}(1540)$ & & & 0.0052 & 0.0045 \\
\hline
\end{tabular}

which mainly differ with regard to the included resonances, their decay modes and branching ratios.

The fitted parameters within the main scheme $\operatorname{SHM}\left(\gamma_{S}\right)$ are shown in Table VII, while the experimental and fitted multiplicities, along with the predicted yields of several had-
TABLE IV. Comparison between measured and fitted particle multiplicities, in the framework of $\operatorname{SHM}\left(\gamma_{S}\right)$ model, in central $\mathrm{Pb}-\mathrm{Pb}$ collisions $(7.2 \%)$ at a beam energy of $80 \mathrm{~A} \mathrm{GeV}$. Also shown are the predicted multiplicities of the main hadron species.

\begin{tabular}{|c|c|c|c|c|}
\hline & Reference & Measurement & Fit A & Fit B \\
\hline$N_{P}$ & [1] & $349 \pm 1 \pm 5$ & 352.0 & 351.5 \\
\hline$\pi^{+}$ & [1] & $446 \pm 5 \pm 22$ & 420.3 & 422.7 \\
\hline$\pi^{-}$ & [1] & $474 \pm 5 \pm 23$ & 450.9 & 453.7 \\
\hline$\pi^{0}$ & & & 485.2 & 457.6 \\
\hline$K^{+}$ & [1] & $76.9 \pm 2 \pm 4$ & 70.72 & 69.87 \\
\hline$K^{-}$ & [1] & $32.4 \pm 0.6 \pm 1.6$ & 35.96 & 35.96 \\
\hline$K_{S}^{0}$ & & & 52.80 & 53.77 \\
\hline$\eta$ & & & 49.37 & 43.47 \\
\hline$\omega$ & & & 39.51 & 34.43 \\
\hline$\phi$ & [22] & $4.37 \pm 0.14$ & 4.354 & 4.353 \\
\hline$\eta^{\prime}$ & & & 3.196 & 2.786 \\
\hline$\rho^{+}$ & & & 47.42 & 48.92 \\
\hline$\rho^{-}$ & & & 51.74 & 53.54 \\
\hline$\rho^{0}$ & & & 50.79 & 52.83 \\
\hline$K^{*+}$ & & & 22.81 & 21.10 \\
\hline$K^{*-}$ & & & 10.43 & 9.600 \\
\hline$K^{* 0}$ & & & 23.28 & 21.73 \\
\hline $\bar{K}^{* 0}$ & & & 9.892 & 9.089 \\
\hline$p$ & & & 141.5 & 142.5 \\
\hline $\bar{p}$ & & & 3.379 & 3.649 \\
\hline$\Delta^{++}$ & & & 30.07 & 28.05 \\
\hline $\bar{\Delta}^{--}$ & & & 0.7623 & 0.7439 \\
\hline$\Lambda$ & [28] & $47.4 \pm 3.7$ & 42.12 & 42.85 \\
\hline $\bar{\Lambda}$ & [28] & $2.26 \pm 0.35$ & 2.171 & 2.328 \\
\hline$\Sigma^{+}$ & & & 11.23 & 10.67 \\
\hline$\Sigma^{-}$ & & & 11.82 & 11.23 \\
\hline$\Sigma^{0}$ & & & 11.56 & 10.93 \\
\hline $\bar{\Sigma}^{-}$ & & & 0.6265 & 0.6348 \\
\hline $\bar{\Sigma}^{+}$ & & & 0.5643 & 0.5729 \\
\hline $\bar{\Sigma}^{0}$ & & & 0.5961 & 0.6024 \\
\hline$\Xi^{-}$ & & & 2.774 & 2.505 \\
\hline$\Xi^{0}$ & & & 2.758 & 2.485 \\
\hline $\bar{\Xi}^{+}$ & & & 0.3279 & 0.3154 \\
\hline$\Xi^{0}$ & & & 0.3428 & 0.3299 \\
\hline$\Omega$ & & & 0.2154 & 0.2090 \\
\hline $\bar{\Omega}$ & & & 0.06132 & 0.06332 \\
\hline$\Lambda(1520)$ & & & 2.769 & 2.028 \\
\hline$\Theta^{+}(1540)$ & & & 2.35 & 2.04 \\
\hline $\bar{\Theta}^{-}(1540)$ & & & 0.022 & 0.021 \\
\hline
\end{tabular}

ron species are shown in Tables I-V and Figs. 1-4. We do not show any plot for the fit in $\mathrm{Pb}-\mathrm{Pb}$ collisions at $30 \mathrm{~A} \mathrm{GeV}$ because of the low number of data points. The quality of the fit is good throughout, as proved by the $\chi^{2}$ 's values and we do not see any clear discrepancy between data and model, 
TABLE V. Comparison between measured and fitted particle multiplicities, in the framework of various versions of the SHM, in central $\mathrm{Pb}-\mathrm{Pb}$ collisions $(5 \%)$ at a beam energy of $158 \mathrm{~A} \mathrm{GeV}$. Also shown are the predicted multiplicities of the main hadron species. The $\Lambda(1520)$ multiplicity was not used in the fits (see text). For the $\operatorname{SHM}\left(\gamma_{S}, \gamma_{q}\right)$ model, the multiplicities are those calculated in the $\gamma_{q}=1.6$ fit.

\begin{tabular}{|c|c|c|c|c|c|c|c|}
\hline Particle & Reference & Measurement & $\operatorname{SHM}\left(\gamma_{S}\right)$ fit $\mathrm{A}$ & $\operatorname{SHM}\left(\gamma_{S}\right)$ fit B & SHM(SCV) & SHM(TC) & $\operatorname{SHM}\left(\gamma_{S}, \gamma_{q}\right)$ \\
\hline$N_{P}$ & {$[1]$} & $362 \pm 1 \pm 5$ & 363.6 & 363.7 & 362.0 & 364.2 & 362.6 \\
\hline$\pi^{+}$ & [1] & $619 \pm 17 \pm 31$ & 551.5 & 533.2 & 502.7 & 563.4 & 578.7 \\
\hline$\pi^{-}$ & [1] & $639 \pm 17 \pm 31$ & 583.5 & 565.6 & 534.1 & 595.3 & 612.5 \\
\hline$\pi^{0}$ & & & 638.2 & 576.2 & 585.4 & 661.9 & 661.6 \\
\hline$K^{+}$ & [1] & $103 \pm 5 \pm 5$ & 103.5 & 103.9 & 106.7 & 99.98 & 102.3 \\
\hline$K^{-}$ & {$[1]$} & $51.9 \pm 1.9 \pm 3$ & 59.57 & 59.35 & 59.54 & 59.23 & 57.77 \\
\hline$K_{S}^{0}$ & {$[28]$} & $81 \pm 4$ & 80.31 & 81.13 & 81.65 & 78.19 & 78.61 \\
\hline$\eta$ & & & 70.69 & 62.90 & 67.73 & 76.94 & 62.22 \\
\hline$\omega$ & & & 54.93 & 45.34 & 48.75 & 58.75 & 43.01 \\
\hline$\phi$ & [29] & $7.6 \pm 1.1$ & 8.136 & 8.676 & 10.07 & 9.088 & 7.084 \\
\hline$\eta^{\prime}$ & & & 4.940 & 4.461 & 5.021 & 5.471 & 3.923 \\
\hline$\rho^{+}$ & & & 64.56 & 61.67 & 57.26 & 67.18 & 54.12 \\
\hline$\rho^{-}$ & & & 69.43 & 66.82 & 61.93 & 72.29 & 57.04 \\
\hline$\rho^{0}$ & & & 68.86 & 66.84 & 61.44 & 73.14 & 57.00 \\
\hline$K^{*+}$ & & & 34.43 & 32.05 & 35.51 & 32.63 & 27.90 \\
\hline$K^{*-}$ & & & 18.10 & 16.47 & 17.79 & 17.61 & 14.79 \\
\hline$K^{* 0}$ & & & 34.93 & 32.81 & 36.12 & 33.07 & 27.99 \\
\hline $\bar{K}^{* 0}$ & & & 17.29 & 15.69 & 16.96 & 16.84 & 14.17 \\
\hline$p$ & & & 143.71 & 142.9 & 138.51 & 147.7 & 144.4 \\
\hline $\bar{p}$ & & & 7.053 & 6.877 & 5.756 & 7.721 & 7.046 \\
\hline$\Delta^{++}$ & & & 31.01 & 28.32 & 29.70 & 30.70 & 29.27 \\
\hline $\bar{\Delta}^{--}$ & & & 1.595 & 1.393 & 1.295 & 1.716 & 1.472 \\
\hline$\Lambda$ & [28] & $53.0 \pm 5.0$ & 53.88 & 56.22 & 57.06 & 49.53 & 53.38 \\
\hline $\bar{\Lambda}$ & [28] & $4.64 \pm 0.32$ & 4.976 & 5.077 & 4.698 & 4.899 & 4.878 \\
\hline$\Sigma^{+}$ & & & 14.45 & 14.11 & 15.28 & 13.21 & 14.57 \\
\hline$\Sigma^{-}$ & & & 15.04 & 14.71 & 15.98 & 13.73 & 14.80 \\
\hline$\Sigma^{0}$ & & & 14.78 & 14.39 & 15.67 & 13.57 & 14.73 \\
\hline $\bar{\Sigma}^{-}$ & & & 1.424 & 1.375 & 1.348 & 1.392 & 1.396 \\
\hline $\bar{\Sigma}^{+}$ & & & 1.301 & 1.256 & 1.224 & 1.277 & 1.288 \\
\hline $\bar{\Sigma}^{0}$ & & & 1.364 & 1.313 & 1.288 & 1.344 & 1.345 \\
\hline$\Xi^{-}$ & [30] & $4.45 \pm 0.22$ & 4.4581 & 4.335 & 4.757 & 4.446 & 4.650 \\
\hline$\Xi^{0}$ & & & 4.446 & 4.315 & 4.736 & 4.440 & 4.681 \\
\hline $\bar{\Xi}^{+}$ & {$[30]$} & $0.83 \pm 0.04$ & 0.8159 & 0.7931 & 0.8234 & 0.8186 & 0.8263 \\
\hline$\Xi^{0}$ & & & 0.8485 & 0.8264 & 0.8578 & 0.8508 & 0.8593 \\
\hline$\Omega$ & {$[22]$} & $0.62 \pm 0.09$ & 0.4499 & 0.4906 & 0.4414 & 0.5165 & 0.4299 \\
\hline $\bar{\Omega}$ & [22] & $0.20 \pm 0.03$ & 0.1702 & 0.1884 & 0.1690 & 0.1859 & 0.1535 \\
\hline$\Lambda(1520)$ & [41] & $1.57 \pm 0.44$ & 3.669 & 2.724 & 3.889 & 3.382 & 3.079 \\
\hline$\Theta^{+}(1540)$ & & & 2.68 & 2.41 & & & 5.71 \\
\hline $\bar{\Theta}^{-}(1540)$ & & & 0.061 & 0.053 & & & 0.13 \\
\hline
\end{tabular}

with the remarkable exception of $\Lambda(1520)$ in $\mathrm{Pb}-\mathrm{Pb}$ collisions at $158 \mathrm{~A} \mathrm{GeV}$. Due to the $5 \sigma$ deviation from the statistical model prediction, the measured $\Lambda(1520)$ yield has been removed from the fitted data sample as it could have biased the fit itself. We argue that this disagreement owes to its short lifetime $(\Gamma=15.6 \mathrm{MeV})$ compared with all other used par- ticles. If the kinetic freeze-out occurs after some suitable delay from the chemical freeze-out, one can indeed justify the low measured $\Lambda(1520)$ yield as the effect of the elastic reinteractions of its decay products. The quality of the fits is further illustrated in Fig. 5 where the measured and fitted ratios $\left\langle\pi^{-}\right\rangle /\left\langle N_{p}\right\rangle$ and $\left\langle K^{+}\right\rangle /\left\langle\pi^{+}\right\rangle$are plotted as a function of 
TABLE VI. Integrated multiplicities over limited rapidity windows around midrapidity obtained by fitting the measured distributions with single $(G)$ or double $(G+G)$ Gaussians. The yields at $10 \%$ and $20 \%$ centrality have been then multiplied by 1.08 and 1.32 , respectively, to convert them at $5 \%$ centrality trigger condition.

\begin{tabular}{ccccccc}
\hline \hline Particle & Centrality $(\%)$ & Reference & Fitting function & $\Delta y=2$ & $\Delta y=1$ & $\chi^{2} /$ dof \\
\hline$\pi^{-}$ & 5 & {$[1]$} & $G+G$ & 333.16 & 176.82 & 9.37 \\
$K^{+}$ & 5 & {$[1]$} & $G+G$ & 57.16 & 29.81 & 3.33 \\
$K^{-}$ & 5 & {$[1]$} & $G+G$ & 32.24 & 16.90 & 1.27 \\
$\phi$ & 5 & {$[29]$} & $G$ & 4.327 & 2.35 & 0.04 \\
$\Lambda$ & 10 & {$[28]$} & $G$ & 22.56 & 11.52 & 0.49 \\
$\bar{\Lambda}$ & 10 & {$[28]$} & $G$ & 3.039 & 1.723 & 0.58 \\
$\Xi^{-}$ & 10 & {$[30]$} & $G$ & 2.75 & 1.484 & 1.2 \\
$\Xi^{+}$ & 10 & {$[30]$} & $G$ & 0.571 & 0.3314 & 0.71 \\
$\Omega$ & 20 & {$[22]$} & $G$ & 0.3095 & 0.173 & 0.73 \\
$\bar{\Omega}$ & 20 & {$[22]$} & $G$ & 0.126 & 0.0789 & 2.70 \\
\hline \hline
\end{tabular}

$\sqrt{s_{N N}}$. These ratios have been chosen as it has been proposed that their energy dependence plays an important role in the search for deconfinement onset at SPS energies [3].

The observed differences in the fit parameters between A and $\mathrm{B}$ are of the order of the fit errors. They may be considered as an estimate of the systematic error due to uncertainties in the implementation of the model. The first set of parameters in Table VII has been obtained by using the full data, while the second set has been obtained by using the maximal common set of particles measured in the four collisions, that is, $\pi^{+}, K^{+}, K^{-}, \Lambda, \bar{\Lambda}$, and the participant nucleons (net baryon number) $B$. By comparing fit results in the same analysis (A with A and B with B), it can be seen that the obtained parameters are in good agreement and only in one case a discrepancy larger than one standard deviation $\left(\gamma_{S}\right.$ in $\mathrm{Pb}-\mathrm{Pb}$ at $158 \mathrm{~A} \mathrm{GeV})$ is observed. This demonstrates the robustness of the results.

We have also included in Tables I-V the prediction for the yield of the recently discovered $\Theta^{+}$pentaquark baryon $(u u d d \bar{s})$ by using as input mass $m=1540 \mathrm{MeV}$ and $J=1 / 2$. According to the $\operatorname{SHM}\left(\gamma_{S}\right)$ model, in the Boltzmann limit this simply reads

$$
\left\langle n_{\Theta^{+}}\right\rangle=\frac{\gamma_{S} V}{\pi^{2}} m^{2} T \mathrm{~K}_{2}\left(\frac{m}{T}\right) \exp \left[\mu_{B} / T+\mu_{Q} / T+\mu_{S} / T\right]
$$

if we disregard feeding from possible excited states.

As the number of data points in $\mathrm{Pb}-\mathrm{Pb}$ collisions at $30 \mathrm{~A} \mathrm{GeV}$ was not sufficient to determine the four free parameters unambiguously, we have forced $T$ to lie on the parabolic chemical freeze-out curve (19) in Sec. V, interpolating the other four points in the $\mu_{B}-T$ plane. This method has proved to be able to provide unambiguous solutions for the remaining three free parameters.

The first quoted error beside the best-fit value in Table VII is the error coming out from the fitting program (inferred from the analysis of the $\chi^{2}=\chi_{\min }^{2}+1$ level contours) whereas the second error is the fit error rescaled by a factor

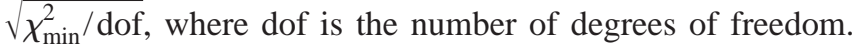

We deem that the latter is a more realistic uncertainty on the parameters with respect to the fit error because of the "imperfect" $\chi_{\min }^{2}$ /dof values, expected to be 1 on average if the model correctly matched physical reality. The argument, which is the same used in the Particle Data Group [21] when averaging discrepant data, is as follows: if $\chi_{\min }^{2} / \mathrm{dof} \neq 1$, then the model cannot reproduce the data at the level of accuracy relevant to the experimental errors; on the other hand, this would be the case if experimental errors were larger and, particularly, if they were rescaled by a common factor $S$ so that

$$
\chi^{2^{\prime}}=\sum_{i} \frac{\left(n_{i}^{\mathrm{exp}}-n_{i}^{\text {theo }}\right)^{2}}{\left(S \sigma_{i}\right)^{2}}=\frac{\chi^{2}}{S^{2}} .
$$

With this simple rescaling of $\chi^{2}$, the best-fit parameters would be unchanged, whereas their relevant errors would scale up by a factor of $S$. In fact, the new covariance matrix $C^{\prime}$ for the parameter vector $X$ is related to the $\chi^{2^{\prime}}$ around the minimum through

$$
\chi^{2^{\prime}}(X)=\chi_{\min }^{2^{\prime}}+\left(X-X_{0}\right)^{T} C^{\prime-1}\left(X-X_{0}\right) .
$$

Also,

$$
\chi^{2}(X)=\chi_{\min }^{2}+\left(X-X_{0}\right)^{T} \mathrm{C}^{-1}\left(X-X_{0}\right)
$$

and, having $\chi^{2^{\prime}}=\chi^{2} / S^{2}$, one finds $\mathrm{C}^{\prime}=S^{2} \mathrm{C}$. Therefore, since this covariance matrix would be the outcome of a standard quality fit, with $\chi_{\min }^{2^{\prime}} / \mathrm{dof}=1$, the errors rescaled by $S$ can be regarded as the sensible minimal uncertainty on the parameters. These rescaled errors have indeed been used in all of the plots in this paper.

A major issue in the multiplicity fits is where to stop the inclusion of heavy light-flavored resonances contributing to measured particle yields in Eq. (3). The relevance of this cutoff is owing to the peculiar shape of the hadron mass spectrum, which rises almost exponentially between 1 and $1.7 \mathrm{GeV}$ and drops thereafter probably due to the missing experimental information (see Fig. 6). Should the number of 
TABLE VII. Summary of fitted parameters in various heavy ion collisions at AGS and SPS in the framework of the $\operatorname{SHM}\left(\gamma_{S}\right)$ model. The "common set" parameters have been obtained by fitting to the measured multiplicities of $\pi^{+}, K^{+}, K^{-}, \Lambda, \bar{\Lambda}$, and the participant nucleons in each collision. Also quoted are minimum $\chi^{2}$ 's, the estimated radius of the EGC, and the $\lambda_{S}$ parameter (see Sec. IV). The rescaled errors (see text) are quoted within brackets. For $\mathrm{Pb}-\mathrm{Pb}$ at $30 \mathrm{~A} \mathrm{GeV}$ of beam energy, we have constrained $T$ and $\mu_{B}$ to lie on the fitted chemical freeze-out curve, as described in Sec. IV.

\begin{tabular}{|c|c|c|c|c|}
\hline Parameters & Main analysis A & Main analysis B & Common set A & Common set B \\
\hline \multicolumn{5}{|c|}{$\mathrm{Au}-\mathrm{Au} 11.6 A \mathrm{GeV}$} \\
\hline$T(\mathrm{MeV})$ & $118.1 \pm 3.5(4.1)$ & $119.1 \pm 4.0(5.4)$ & $119.2 \pm 2.1(2.9)$ & $119.1 \pm 4.0(6.6)$ \\
\hline$\mu_{B}(\mathrm{MeV})$ & $555 \pm 12(13)$ & $578 \pm 15(21)$ & $556 \pm 12(17)$ & $576.9 \pm 17.2(29)$ \\
\hline$\gamma_{S}$ & $0.652 \pm 0.069(0.079)$ & $0.763 \pm 0.086(0.12)$ & $0.645 \pm 0.042(0.058)$ & $0.761 \pm 0.090(0.15)$ \\
\hline$V T^{3} \exp [-0.7 \mathrm{GeV} / T]$ & $1.94 \pm 0.21(0.24)$ & $1.487 \pm 0.18(0.25)$ & $1.97 \pm 0.12(0.17)$ & $1.494 \pm 0.21(0.35)$ \\
\hline$\chi^{2} /$ dof & $4.0 / 3$ & $5.5 / 3$ & $3.86 / 2$ & $5.5 / 2$ \\
\hline$R(\mathrm{fm})$ & $9.31 \pm 0.69(0.80)$ & $8.32 \pm 0.72(0.97)$ & & \\
\hline \multirow[t]{2}{*}{$\lambda_{S}$} & $0.381 \pm 0.053(0.061)$ & $0.490 \pm 0.084(0.11)$ & $0.401 \pm 0.053(0.074)$ & $0.487 \pm 0.089(0.15)$ \\
\hline & & $\mathrm{Pb}-\mathrm{Pb} 30 A \mathrm{GeV}$ & & \\
\hline$T(\mathrm{MeV})$ & 139.5 & 140.3 & & \\
\hline$\mu_{B}(\mathrm{MeV})$ & 428.6 & 428.7 & & \\
\hline \multirow{5}{*}{$\begin{array}{c}\gamma_{S} \\
V T^{3} \exp [-0.7 \mathrm{GeV} / T] \\
\chi^{2} / \text { dof } \\
\lambda_{S}\end{array}$} & $0.938 \pm 0.078(0.13)$ & $1.051 \pm 0.103(0.16)$ & & \\
\hline & $6.03 \pm 0.50(0.85)$ & $5.273 \pm 0.526(0.80)$ & & \\
\hline & $5.75 / 2$ & $4.6 / 2$ & & \\
\hline & $0.611 \pm 0.037(0.062)$ & $0.683 \pm 0.086(0.13)$ & & \\
\hline & & $\mathrm{Pb}-\mathrm{Pb} 40 A \mathrm{GeV}$ & & \\
\hline$T(\mathrm{MeV})$ & $147.6 \pm 2.1(4.0)$ & $145.5 \pm 1.9(3.5)$ & $148.6 \pm 2.1(4.7)$ & $146.1 \pm 2.0(4.0)$ \\
\hline$\mu_{B}(\mathrm{MeV})$ & $380.3 \pm 6.5(13)$ & $375.4 \pm 6.4(12)$ & $393 \pm 10(22)$ & $390 \pm 10(21)$ \\
\hline & $0.757 \pm 0.024(0.046)$ & $0.807 \pm 0.025(0.047)$ & $0.874 \pm 0.064(0.14)$ & $0.961 \pm 0.079(0.16)$ \\
\hline \multirow{5}{*}{$\begin{array}{c}V T^{3} \exp [-0.7 \mathrm{GeV} / T] \\
\chi^{2} / \mathrm{dof} \\
R(\mathrm{fm}) \\
\lambda_{S}\end{array}$} & $8.99 \pm 0.37(0.71)$ & $8.02 \pm 0.34(0.63)$ & $8.09 \pm 0.55(1.24)$ & $7.08 \pm 0.53(1.1)$ \\
\hline & $14.7 / 4$ & $13.6 / 4$ & $10.1 / 2$ & $8.1 / 2$ \\
\hline & $8.37 \pm 0.32(0.61)$ & $8.37 \pm 0.31(0.58)$ & & \\
\hline & $0.507 \pm 0.025(0.049)$ & $0.505 \pm 0.026(0.048)$ & $0.621 \pm 0.064(0.14)$ & $0.626 \pm 0.071(0.14)$ \\
\hline & & $\mathrm{Pb}-\mathrm{Pb} 80 A \mathrm{GeV}$ & & \\
\hline$T(\mathrm{MeV})$ & $153.7 \pm 2.8(4.7)$ & $151.9 \pm 3.4(5.4)$ & $154.6 \pm 3.3(7.2)$ & $152.2 \pm 3.5(7.5)$ \\
\hline$\mu_{B}(\mathrm{MeV})$ & $297.7 \pm 5.9(9.8)$ & $288.9 \pm 6.8(11)$ & $300.7 \pm 9.4(21)$ & $292.8 \pm 9.0(19)$ \\
\hline \multirow{6}{*}{$\begin{array}{c}\gamma_{S} \\
V T^{3} \exp [-0.7 \mathrm{GeV} / T] \\
\chi^{2} / \mathrm{dof} \\
R(\mathrm{fm}) \\
\lambda_{S}\end{array}$} & $0.730 \pm 0.021(0.035)$ & $0.766 \pm 0.026(0.042)$ & $0.741 \pm 0.057(0.13)$ & $0.782 \pm 0.061(0.13)$ \\
\hline & $15.38 \pm 0.61(1.0)$ & $14.12 \pm 0.65(1.1)$ & $15.0 \pm 1.0(2.3)$ & $13.7 \pm 0.95(2.0)$ \\
\hline & $11.0 / 4$ & $10.4 / 4$ & $9.6 / 2$ & $9.3 / 2$ \\
\hline & $9.03 \pm 0.41(0.68)$ & $9.05 \pm 0.44(0.71)$ & & \\
\hline & $0.455 \pm 0.020(0.034)$ & $0.461 \pm 0.020(0.032)$ & $0.482 \pm 0.053(0.12)$ & $0.4568 \pm 0.044(0.095)$ \\
\hline & & $\mathrm{Pb}-\mathrm{Pb} 158 A \mathrm{GeV}$ & & \\
\hline$T(\mathrm{MeV})$ & $157.8 \pm 1.4(1.9)$ & $154.8 \pm 1.4(2.1)$ & $156.6 \pm 2.3(3.3)$ & $152.7 \pm 2.1(3.2)$ \\
\hline$\mu_{B}(\mathrm{MeV})$ & $247.3 \pm 5.2(7.2)$ & $244.5 \pm 5.0(7.8)$ & $238.6 \pm 7.1(10)$ & $232.4 \pm 7.7(12)$ \\
\hline$\gamma_{S}$ & $0.843 \pm 0.024(0.033)$ & $0.938 \pm 0.027(0.042)$ & $0.722 \pm 0.053(0.077)$ & $0.764 \pm 0.065(0.097)$ \\
\hline$V T^{3} \exp [-0.7 \mathrm{GeV} / T]$ & $21.13 \pm 0.80(1.1)$ & $18.46 \pm 0.69(1.1)$ & $23.2 \pm 1.4(2.0)$ & $21.1 \pm 1.4(2.2)$ \\
\hline$\chi^{2} /$ dof & $16.9 / 9$ & $21.6 / 9$ & $4.2 / 2$ & $4.5 / 2$ \\
\hline$R(\mathrm{fm})$ & $9.41 \pm 0.26(0.35)$ & $9.44 \pm 0.25(0.39)$ & & \\
\hline$\lambda_{S}$ & $0.506 \pm 0.018(0.024)$ & $0.514 \pm 0.018(0.028)$ & $0.426 \pm 0.037(0.054)$ & $0.401 \pm 0.039(0.058)$ \\
\hline
\end{tabular}

states keep on increasing exponentially, the problem is a set of the physical meaning of the obtained parameters, which could be heavily affected by the ignorance of further hadronic states. In fact, although the production of resonances decreases exponentially with the mass, the effect on second- ary light particles through the decay chain could be balanced and even exceeded by the increasing number of states. We have thus checked the stability of the obtained parameters in the four collisions by varying the cutoff on the mass spectrum in a range where we are reasonably confident of the 

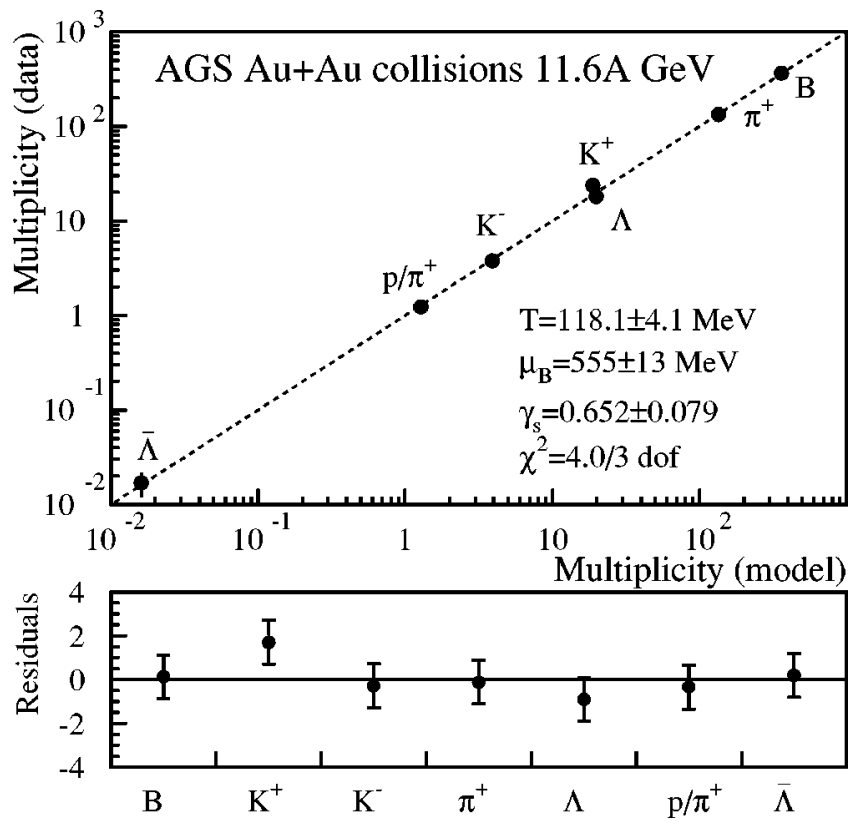

FIG. 1. Above: measured vs fitted multiplicities in the statistical model supplemented with $\gamma_{S}$ parameter $\left[\operatorname{SHM}\left(\gamma_{S}\right)\right]$ in $\mathrm{Au}-\mathrm{Au}$ collisions at a beam energy of $11.6 \mathrm{~A} \mathrm{GeV}$ in the fit $\mathrm{A}$; also quoted are the best-fit parameters. Below: residual distribution.

complete experimental knowledge and the number of states apparently rises exponentially (i.e., up to $1.7-1.8 \mathrm{GeV}$ ) and by repeating the fit. As shown in Fig. 7, the fitted $T, \mu_{B}$, and $\gamma_{S}$ in $\mathrm{Pb}-\mathrm{Pb}$ at $158 \mathrm{~A} \mathrm{GeV}$ are indeed fairly constant from 1.3 to $1.9 \mathrm{GeV}$. Furthermore, the outcoming primary yields of some measured particles tend to saturate at cutoff masses of about $1.8 \mathrm{GeV}$, implying that the contribution of resonance

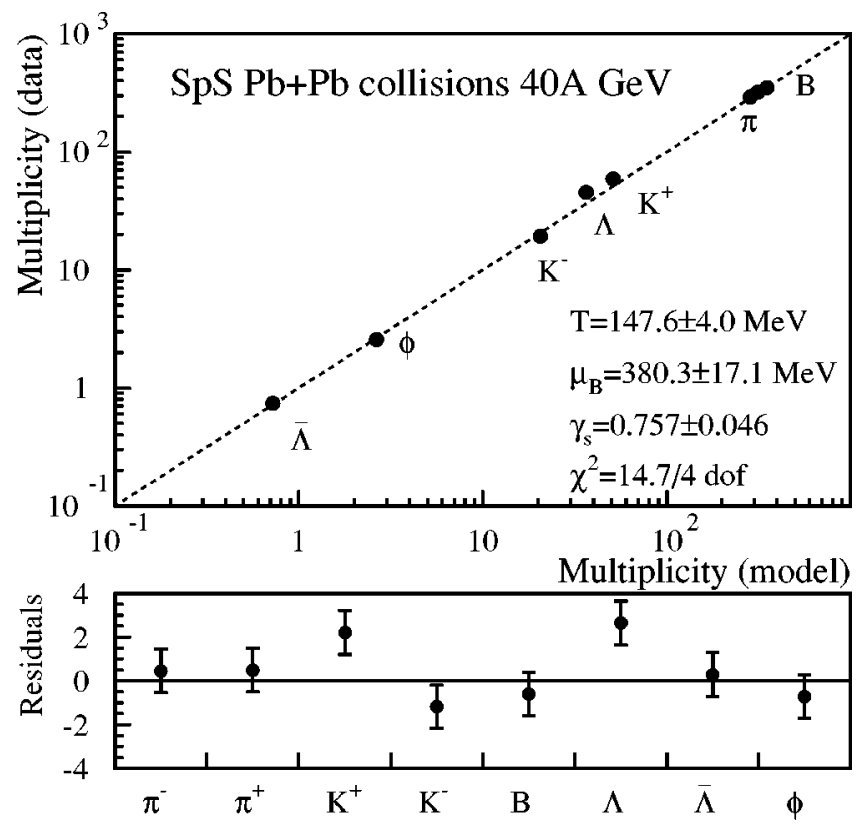

FIG. 2. Above: measured vs fitted multiplicities in the statistical model supplemented with $\gamma_{S}$ parameter in $\mathrm{Pb}-\mathrm{Pb}$ collisions at a beam energy of $40 \mathrm{~A} \mathrm{GeV}$ in the fit $\mathrm{A}$; also quoted are the best-fit parameters. Below: residual distribution.

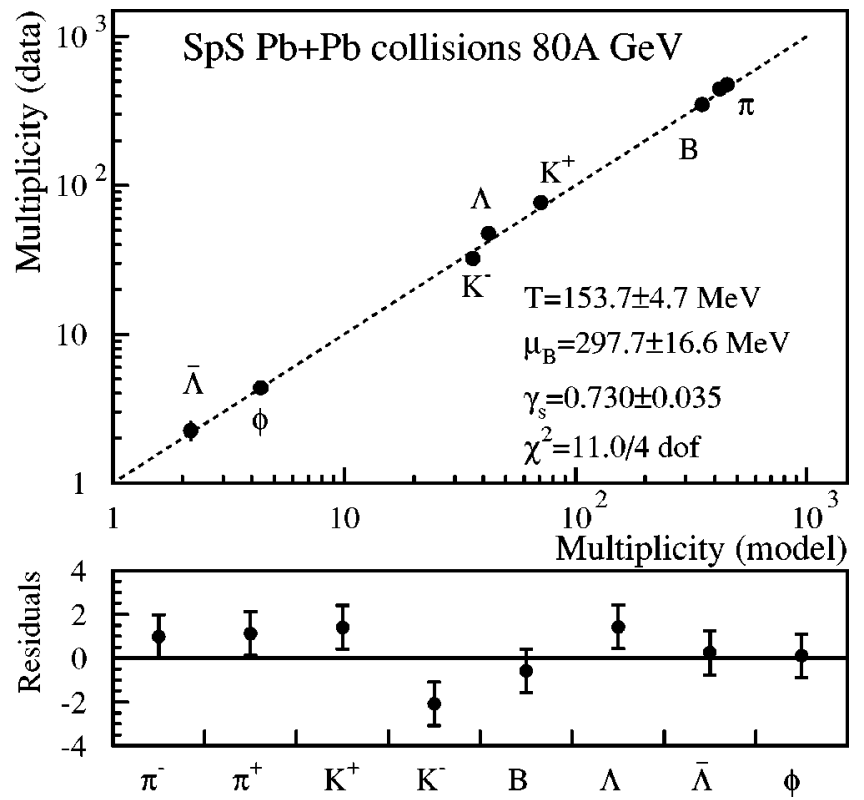

FIG. 3. Above: measured vs fitted multiplicities in the statistical model supplemented with $\gamma_{S}$ parameter in $\mathrm{Pb}-\mathrm{Pb}$ collisions at a beam energy of $80 \mathrm{~A} \mathrm{GeV}$ in the fit A; also quoted are the best-fit parameters. Below: residual distribution.

decays to secondaries (needed to keep the final multiplicity close to the measured value) settles down as well and the inclusion of heavier states yields a more and more negligible contribution. This is a clear indication of the significance of the fit results. A similar pattern occurs in all other examined collisions.

A major result of these fits is that $\gamma_{S}$ is significantly

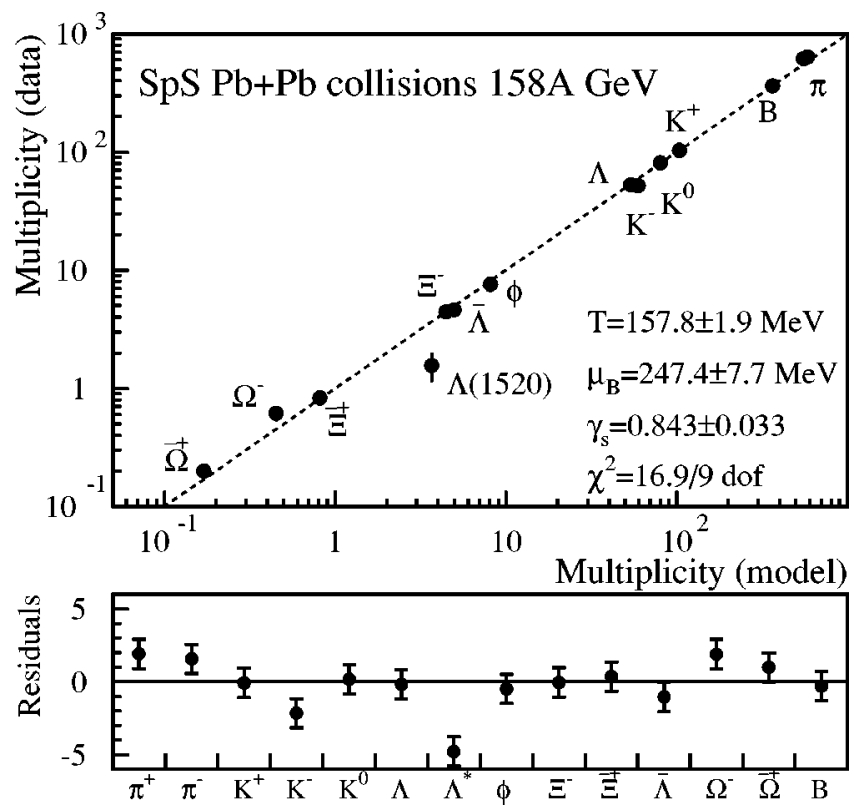

FIG. 4. Above: measured vs fitted multiplicities in the statistical model supplemented with $\gamma_{S}$ parameter in $\mathrm{Pb}-\mathrm{Pb}$ collisions at a beam energy of $158 \mathrm{~A} \mathrm{GeV}$ in the fit A; also quoted are the best-fit parameters. Below: residual distribution. Note that $\Lambda(1520)$ was not used in the fit (see text). 


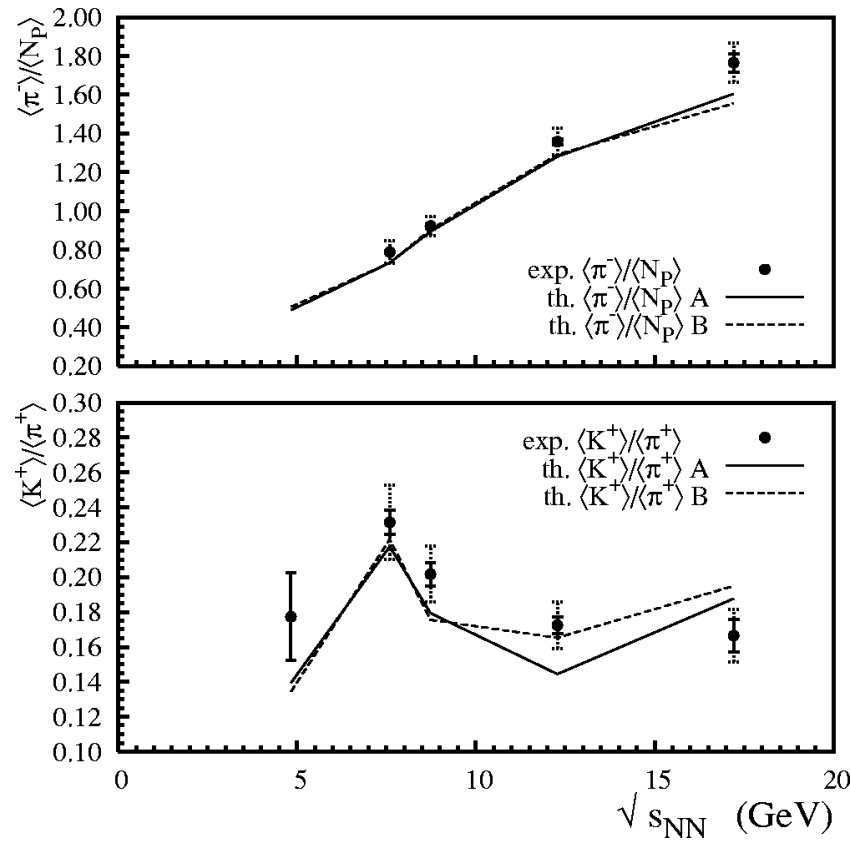

FIG. 5. Comparison between measured and calculated (in fits A and B) $\left\langle K^{+}\right\rangle /\left\langle\pi^{+}\right\rangle$and $\left\langle\pi^{-}\right\rangle /\left\langle\mathrm{N}_{p}\right\rangle$ ratios as a function of the centerof-mass energy in the examined collisions. For the SPS energy points the statistical errors are indicated with solid lines, while the contribution of the common systematic error is shown as a dotted line. The lines connect the fitted values.

smaller than 1 in almost all cases (with a possible exception at $30 \mathrm{~A} \mathrm{GeV}$, see Table VII), that is, strangeness seems to be undersaturated with respect to a completely chemically equilibrated hadron gas. This confirms previous findings $[6,9,27]$. There is a considerable interest and ongoing investigations about this deviation of the data from the fully equilibrated hadron gas, particularly motivated by the fact

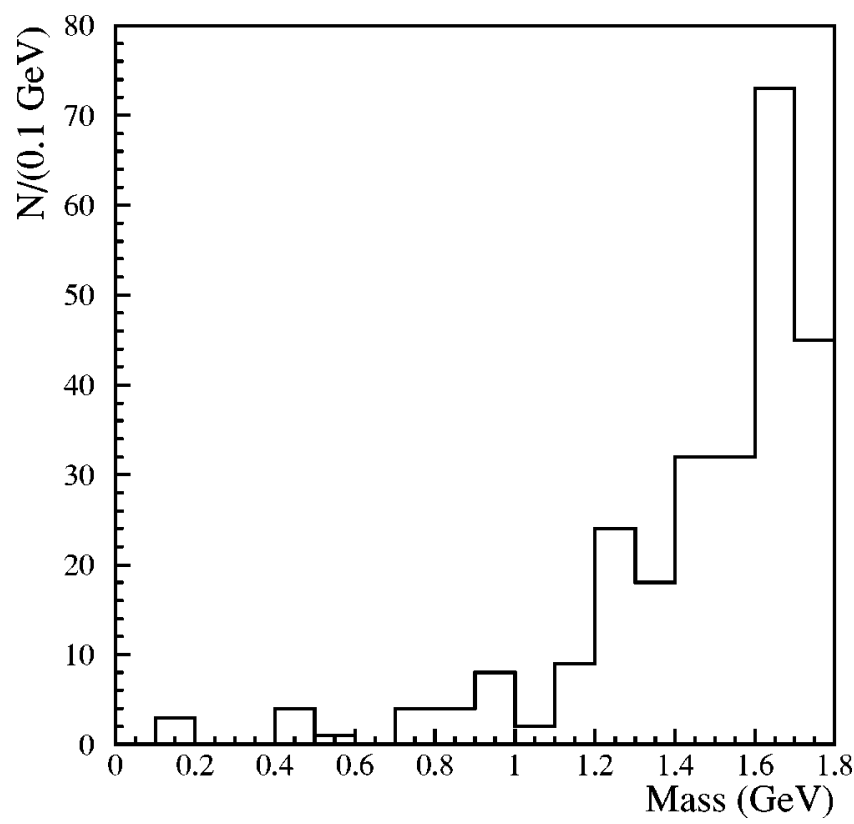

FIG. 6. Spectrum of known light-flavored hadronic species up to a mass of $1.8 \mathrm{GeV}$.

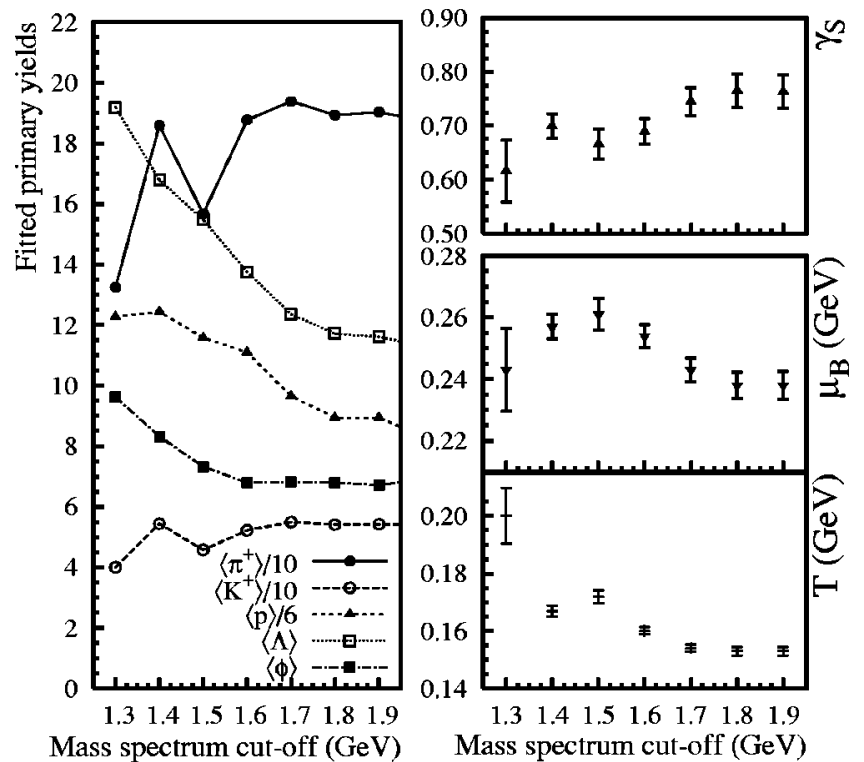

FIG. 7. Left: primary yields of various particles as a function of the cutoff on the hadronic mass spectrum. Right: fitted $\gamma_{S}$, baryonchemical potential, and temperature as a function of the cutoff on the hadronic mass spectrum.

that strangeness production is considered as a possible QGP signature. It is therefore worth examining and testing, with the presently available large data sample, different scenarios which have been put forward to account for the undersaturation of strangeness.

\section{A. Full equilibrium and midrapidity ratios}

As we have seen, fits to full phase space multiplicities within the SHM yield $\gamma_{S}<1$ in most cases. However, good tests of the same model without extra strangeness suppression (i.e., assuming $\gamma_{S}=1$ ) have been obtained by fitting ratios of hadronic yields within a limited rapidity range around midrapidity at top SPS energy [7]. This is an appropriate method of estimating the parameters of the sources only if the boost-invariant Bjorken scenario holds, at least as a good approximation, over a large rapidity interval (say $\Delta y \simeq 6$ ) because, in this case, particle ratios at midrapidity are the same as in full phase space. However, rapidity distributions of hadrons at SPS energies do not feature boost invariance $[1,22,28-30]$ and a cut at midrapidity can artificially enhance heavy particle yield with respect to light ones (see Fig. 8), as long as their kinetic freeze-out occurs at the same temperature and the leading baryon effect can be neglected. In the statistical model of a single fireball this can be easily understood, for the width of the rapidity distribution decreases as a function of mass according to (in the Boltzmann approximation)

$$
\frac{d N}{d y} \propto\left(m^{2} T+\frac{2 m T^{2}}{\cosh y}+\frac{2 T^{3}}{\cosh ^{2} y}\right) \exp [-m \cosh y / T] .
$$

Yet, it is worth testing the effect of the rapidity cut on measured distributions rather than using arguments based solely on the statistical model. Therefore, we have fitted, within the 


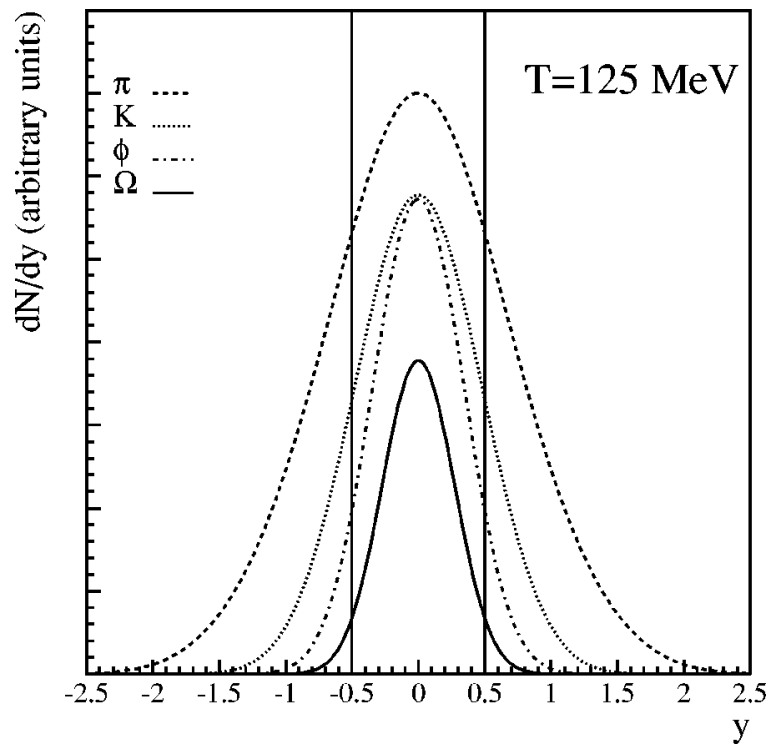

FIG. 8. Rapidity distributions of $\pi^{ \pm}, K^{ \pm}, \phi$, and $\Omega$ emitted from a single fireball at rest at a kinetic freeze-out temperature of $T$ $=125 \mathrm{MeV}$.

scheme $\mathrm{A}$, the integrated yields over limited rapidity windows measured in central $\mathrm{Pb}+\mathrm{Pb}$ collisions at $158 \mathrm{~A} \mathrm{GeV}$ by NA49 (see Sec. III for details) and quoted in Table VIII as well as the yields measured by WA97 [31] over a $\Delta y=1$ window around midrapidity. We first note that, according to Table VIII, the integrated yields over $\Delta y=1$ measured by NA49 and WA97 are in good agreement with each other. Since the fit to the statistical model gave $\gamma_{S} \simeq 1$ [32] for WA97 data, the same is expected for the integrated NA49 yields over the same rapidity window. This is indeed what we find, as shown in Table IX. While temperature and baryon-chemical potential are essentially unchanged, the best-fit value of $\gamma_{S}$ is closer to 1 than that obtained in full phase space in fit A (see Table VII) and it is also compatible with 1 within the error.

We then conclude that the superfluity of $\gamma_{S}$ in analysis of midrapidity particle yields, at least at top SPS energy, is likely owing to the artificial enhancement of strange particles with respect to lighter nonstrange ones, induced by the cut on rapidity. The fact that $\gamma_{S} \simeq 1$ for midrapidity yields is then not an indication of a fully equilibrated hadron gas at midrapidity; even if such equilibrated fireball existed at the estimated kinetic freeze-out temperature of $T \approx 125 \mathrm{MeV}$ [33], the $\Delta y=1$ window would be too narrow for a correct estimation of chemical freeze-out parameters (see Fig. 8) because lighter particles would be cut down significantly.
TABLE VIII. Fit results in $\mathrm{Pb}-\mathrm{Pb}$ at $158 \mathrm{~A} \mathrm{GeV}$ with particle yields in limited rapidity window.

\begin{tabular}{ccccc}
\hline \hline Particle & $\begin{array}{c}\text { WA97 } \\
\text { measured }\end{array}$ & $\begin{array}{c}\text { WA97 } \\
\text { fitted }\end{array}$ & $\begin{array}{c}\text { NA49 } \\
\text { measured }\end{array}$ & $\begin{array}{c}\text { NA49 } \\
\text { fitted }\end{array}$ \\
\hline$h^{-}$ & $178 \pm 22$ & 157.2 & & \\
$\pi^{-}$ & & & $176.8 \pm 9.8$ & 151.8 \\
$K^{+}$ & & & $29.81 \pm 2.05$ & 30.63 \\
$K^{-}$ & & & $16.90 \pm 1.16$ & 18.24 \\
$K_{S}^{0}$ & $21.9 \pm 2.4$ & 22.97 & & \\
$\phi$ & & & $2.35 \pm 0.34$ & 2.900 \\
$\Lambda$ & $13.7 \pm 0.9$ & 13.75 & $12.44 \pm 1.17$ & 15.35 \\
$\bar{\Lambda}$ & $1.8 \pm 0.2$ & 1.837 & $1.86 \pm 0.13$ & 1.905 \\
$\Xi^{-}$ & $1.5 \pm 0.1$ & 1.525 & $1.603 \pm 0.079$ & 1.502 \\
$\bar{\Xi}^{+}$ & $0.37 \pm 0.06$ & 0.3782 & $0.358 \pm 0.017$ & 0.3585 \\
$\Omega$ & & & $0.228 \pm 0.033$ & 0.1858 \\
$\bar{\Omega}$ & & & $0.104 \pm 0.016$ & 0.08894 \\
$\Omega+\bar{\Omega}$ & $0.41 \pm 0.08$ & 0.3136 & & \\
\hline \hline
\end{tabular}

\section{B. Strangeness correlation volume}

To account for the observed undersaturation of strangeness, a picture has been put forward in which strangeness is supposed to be exactly vanishing over distances less than those implied by the overall volume $V$ [34]. We henceforth refer to this version of the statistical model as SHM(SCV) (strangeness correlation volume). Following the description of the model in Sec. II, this means that the produced clusters or fireballs emerge with $S=0$ and they are not allowed to share nonvanishing net strangeness. Assuming, for sake of simplicity, that all clusters have the same typical volume $V_{c}$ and that the equivalence of the set of clusters to a global fireball still applies for baryon number and electric charge (but not to strangeness) the following expression of the primary average multiplicities can be obtained (see Appendix B):

$$
\begin{aligned}
\left\langle n_{j}\right\rangle= & \frac{V}{V_{c}} \frac{\left(2 J_{j}+1\right) V_{c}}{(2 \pi)^{3}} \sum_{n=1}^{\infty} \int d^{3} p(\mp 1)^{n+1} \\
& \times \exp \left[-n \sqrt{p^{2}+m_{j}^{2}} / T+n \mu_{B} B_{j} / T\right. \\
& \left.+n \mu_{Q} Q_{j} / T\right] \frac{Z_{c}\left(-n S_{j}\right)}{Z_{c}(0)},
\end{aligned}
$$

where

TABLE IX. Fit results in $\mathrm{Pb}-\mathrm{Pb}$ at $158 \mathrm{~A} \mathrm{GeV}$ with particle yields in limited rapidity window.

\begin{tabular}{cccc}
\hline \hline Parameters & $\Delta y=1$ & $\Delta y=2$ & WA97 central \\
\hline$T(\mathrm{MeV})$ & $162.7 \pm 2.7(5.1)$ & $161.0 \pm 2.6(4.2)$ & $161.3 \pm 5.4$ \\
$\mu_{B}(\mathrm{MeV})$ & $229 \pm 12(23)$ & $223 \pm 13(21)$ & $218 \pm 19$ \\
$\gamma_{S}$ & $0.971 \pm 0.044(0.083)$ & $0.950 \pm 0.043(0.070)$ & $1.085 \pm 0.079$ \\
$V T^{3} \exp [-0.7 \mathrm{GeV} / T]$ & $5.55 \pm 0.31(0.58)$ & $10.71 \pm 0.59(0.96)$ & $4.73 \pm 0.52$ \\
$\chi^{2} /$ dof & $21.1 / 6$ & $16.0 / 6$ & $2.7 / 3$ \\
\hline \hline
\end{tabular}


TABLE X. Fit results in $\mathrm{Pb}-\mathrm{Pb}$ at $158 \mathrm{~A} \mathrm{GeV}$ with different models, as described in the text: SHM(SCV) (strangeness correlation volume), $\operatorname{SHM(TC)~(two-component~model),~} \operatorname{SHM}\left(\gamma_{S}, \gamma_{q}\right)$ (light quark nonequilibrium model). Free fit parameters are quoted along with resulting minimum $\chi^{2}$ s. The rescaled errors (see text) are quoted within brackets. For the $\operatorname{SHM}\left(\gamma_{S}, \gamma_{q}\right)$, the fit has been done by fixing $\gamma_{q}=1.6$ near the absolute $\chi^{2}$ minimum (see Sec. IV B).

\begin{tabular}{cccc}
\hline \hline Parameters & $\operatorname{SHM}(\mathrm{SCV})$ & $\operatorname{SHM}(\mathrm{TC})$ & $\operatorname{SHM}\left(\gamma_{S}, \gamma_{q}\right)$ \\
\hline$T(\mathrm{MeV})$ & $157.9 \pm 1.6(3.3)$ & $154.8 \pm 1.5(1.9)$ & $140.4 \pm 1.1(1.3)$ \\
$\mu_{B}(\mathrm{MeV})$ & $261.5 \pm 2.4(4.9)$ & $237.1 \pm 7.0(8.6)$ & $218.1 \pm 4.3(5.2)$ \\
$\gamma_{S}$ & 1.0 (Fixed) & 1.0 (Fixed) & $0.929 \pm 0.027(0.033)$ \\
$V T^{3} \exp [-0.7 \mathrm{GeV} / T]$ & $10.62 \pm 0.52(1.1)$ & $15.50 \pm 0.54(0.67)$ & $16.82 \pm 0.59(0.72)$ \\
$f$ & $0.0253 \pm 0.0067(0.014)$ & & 1.6 (Fixed) \\
$\gamma_{q}$ & & $52.0 \pm 7.8(9.6)$ & \\
$\left\langle N_{c}\right\rangle$ & & $13.7 / 9$ & $13.4 / 9$ \\
$\chi^{2} /$ dof & $37.2 / 9$ &
\end{tabular}

$$
\begin{aligned}
Z_{c}= & \frac{1}{2 \pi} \int_{-\pi}^{\pi} d \phi \exp \left[\sum_{j} \frac{\left(2 J_{j}+1\right) V_{c}}{(2 \pi)^{3}}\right. \\
& \times \int d^{3} p \ln \left(1 \pm e^{\left.-\sqrt{p^{2}+m_{j}^{2}} / T+\mu_{B} B_{j} / T+\mu_{Q} Q_{j} / T-\mathrm{i} \phi S_{j}\right)^{ \pm 1}}\right]
\end{aligned}
$$

is the so-called strange canonical partition function of a single cluster. As usual, in the above equations, the upper sign is for fermions and the lower for bosons.

If $V_{c}$ is sufficiently small, the multiplicities of strange hadrons turn out to be significantly suppressed with respect to the corresponding grand-canonical ones due to the enforcement of exact strangeness conservation in a finite system, an effect called canonical suppression. Furthermore, the suppression features hierarchy in strangeness, namely, it is stronger for $\Omega(S=3)$ and $\Xi(S=2)$ than for kaons and $\Lambda$ 's, so it can be argued that this can account for the actually observed hierarchical pattern of extra strangeness suppression which goes like $\gamma_{S}^{|S|}$ for open strange particles. The discriminating difference between this picture and our main scheme $\operatorname{SHM}\left(\gamma_{S}\right)$ described in Sec. II is concerned with hidden strange particles such as $\phi$, which do not suffer canonical suppression, so that its theoretical multiplicity in SHM(SCV) turns out to be simply the same as in a cluster with volume $V$, that is given by the formula (1) without $\gamma_{S}^{2}$ suppression.

We have made a test of this model by fitting the data sample of full phase space multiplicities in $\mathrm{Pb}-\mathrm{Pb}$ collisions at $158 \mathrm{~A} \mathrm{GeV}$ fixing $\gamma_{S}=1$ and determining the parameters $T$, $V, \mu_{B}$, and $f=V_{c} / V$ within the scheme A. The results are shown in Tables $\mathrm{X}$ and $\mathrm{V}$. The quality of the fit is worse with respect to the $\operatorname{SHM}\left(\gamma_{S}\right)$ model mainly because of the underestimated pion yield and the larger of $\phi$. The latter is expected, as has been mentioned. As far as pion discrepancy is concerned, the deviation stems from the very fact that they are the only nonstrange particles in the fit. The minimization procedure tries to accommodate the relative ratios among strange hadrons by fixing $V_{c}$ and $T$, then it tries to set the overall normalization $V$ and at this stage a competition sets in between the set of strange and nonstrange particles. Since pions are only two entries, the fit prefers to keep them low rather than raising the whole set of strange particles.
Our result suggests that, for the local strangeness correlation to be an effective mechanism, the cluster volume should be of the order of $2.5 \%$ of the overall volume. Otherwise stated, strange quarks should have not propagated beyond a distance of about $4 \mathrm{fm}$ from the production point up to chemical freeze-out, if we take the overall volume of about $3 \times 10^{3} \mathrm{fm}^{3}$ as coming out from this fit where hadrons are pointlike particles.

\section{Superposition of NN collisions with a fully equilibrated fireball}

In this picture, henceforth referred to as $\mathrm{SHM}$ (TC) (two component), the observed hadron production is approximately the superposition of TC's: one originated from one large fireball at complete chemical equilibrium at freeze-out, with $\gamma_{S}=1$, and another component from single nucleonnucleon collisions. In fact, according to simulations based on transport models, a significant fraction of beam nucleons interacts only once with target nucleons [35]. With the simplifying assumption of disregarding subsequent inelastic collisions of particles produced in those primary NN collisions, the overall hadron multiplicity can be written then as

$$
\left\langle n_{j}\right\rangle=\left\langle N_{c}\right\rangle\left\langle n_{j}\right\rangle_{N N}+\left\langle n_{j}\right\rangle_{V},
$$

where $\left\langle n_{j}\right\rangle_{N N}$ is the average multiplicity of the $j$ th hadron in a single NN collision, $\left\langle N_{c}\right\rangle$ is the mean number of single $\mathrm{NN}$ collisions giving rise to nonreinteracting particles, and $\left\langle n_{j}\right\rangle_{V}$ is the average multiplicity of hadrons emitted from the equilibrated fireball, as in Eq. (1), with $\gamma_{S}=1$. The $\left\langle n_{j}\right\rangle_{N N}$ term can be written, in turn, as

$$
\left\langle n_{j}\right\rangle_{N N}=\frac{Z^{2}}{A^{2}}\left\langle n_{j}\right\rangle_{p p}+\frac{(A-Z)^{2}}{A^{2}}\left\langle n_{j}\right\rangle_{n n}+\frac{2 Z(A-Z)}{A^{2}}\left\langle n_{j}\right\rangle_{n p} .
$$

Since it is known that in NN collisions strangeness is strongly suppressed [13] the idea is to ascribe the observed undersaturation of strangeness in heavy ion collisions to the NN component, leaving the central fireball at complete equilibrium, i.e., with $\gamma_{S}=1$. Of course, this is possible provided 
that $\left\langle N_{c}\right\rangle$ is sufficiently large. This production mechanism has probably some consequences on the final rapidity and momentum distributions of the different species, whose calculation goes certainly beyond the scope of this paper. Instead, we have confined ourselves to integrated multiplicities and tried to fit $T, V, \mu_{B}$ of the central fireball and $\left\langle N_{c}\right\rangle$ by using NA49 data in $\mathrm{Pb}-\mathrm{Pb}$ collisions at $158 \mathrm{~A} \mathrm{GeV}$ within the scheme A.

To calculate $\left\langle n_{j}\right\rangle_{N N}$ we have used the statistical model and fitted $p p$ full phase space multiplicities measured at $\sqrt{s}$ $=17.2 \mathrm{GeV}$ (i.e., the same beam energy) by the same NA49 experiment. For $n p$ and $n n$ collisions, the parameters of the statistical model determined in $p p$ are retained and the initial quantum numbers are changed accordingly. Theoretical multiplicities have been calculated in the canonical ensemble, which is described in detail in Ref. [14]. Instead of the usual $\gamma_{S}$ parametrization, the new parametrization described in Ref. [14] has been used in which one assumes that some number of $s \bar{s}$ pairs, Poissonianly distributed, hadronizes; the extra strangeness-suppression parameter $\gamma_{S}$ is thus replaced by the mean number of these $s \bar{s}$ pairs, $\langle s \bar{s}\rangle$.

The results of the fit are shown in Table XI along with fitted and predicted hadron multiplicities, including the $\Theta(1540)$ pentaquark baryon, and in Fig. 9. The temperature value is significantly higher than in $p p$ and $p \bar{p}$ collisions at higher energy, an effect already observed for center-of-mass energies below $20 \mathrm{GeV}[13,14]$. We conjecture that this is a possible indication of a beginning inadequacy of the canonical ensemble at low energy, where exact conservation of energy and momentum should start to play a significant role. Perhaps this is the point where the microcanonical hadronization of each cluster is a more appropriate approach.

The results of the fit to $\mathrm{Pb}-\mathrm{Pb}$ collisions are shown in Table X. The fit quality, as well as the obtained values of $T$, $\mu_{B}$, is comparable to the main fit within the $\operatorname{SHM}\left(\gamma_{S}\right)$ model. The predicted number of "single" NN collisions is about 50 with a $16 \%$ uncertainty. Thus, only 260 nucleons out of 360 contribute to the formation of large equilibrated fireballs. The percentage of primary hadrons stemming from $\mathrm{NN}$ collisions varies from $14 \%$ for pions to $27 \%$ of $\rho$ 's and protons and to $0.5 \%$ of $\Omega$ 's. It should be pointed out that the fitted parameters are affected by a further systematic error owing to the uncertainty on the parameters of the statistical model in NN collisions, which are used as an input in the $\mathrm{Pb}-\mathrm{Pb}$ fit. However, because of exceeding computing time needs, it has not been possible to assess these errors.

In a simple geometrical picture, the single-interacting nucleons are located in the outer corona of the portion of colliding nuclei corresponding to the observed number of participants. As the projected (on the collision's transverse plane) radial nucleon density is

$$
\frac{d N}{d r}=4 \pi r \sqrt{R^{2}-r^{2}} n_{0},
$$

where $n_{0}=0.16 \mathrm{fm}^{-3}$ is the nucleon density and $R$ $\simeq 6.45 \mathrm{fm}$ is the radius of the portion of colliding nucleus corresponding to a participant number of 180 , the 50 single-interacting nucleons should lie between 4.84 and
TABLE XI. Fitted parameters and multiplicities in $p p$ collisions at a beam energy of $158 \mathrm{GeV}$, corresponding to $\sqrt{s}=17.2 \mathrm{GeV}$. The rescaled errors (see text) are quoted within brackets.

\begin{tabular}{|c|c|c|c|}
\hline Parameter & & Value & \\
\hline$T(\mathrm{MeV})$ & & $187.2 \pm 6.1(9.3)$ & \\
\hline$V T^{3}$ & & $5.79 \pm 0.85(1.3)$ & \\
\hline$\langle\overline{\mathrm{ss}}\rangle$ & & $0.381 \pm 0.021(0.032)$ & \\
\hline$\chi^{2} / \mathrm{dof}$ & & $16.1 / 7$ & \\
\hline$\lambda_{S}$ & & $0.224 \pm 0.019(0.024)$ & \\
\hline Particle & Reference & Measurement & Fit \\
\hline$\pi^{+}$ & {$[42]$} & $3.15 \pm 0.06$ & 3.257 \\
\hline$\pi^{-}$ & {$[42]$} & $2.45 \pm 0.12$ & 2.441 \\
\hline$\pi^{0}$ & & & 3.317 \\
\hline$K^{+}$ & {$[42]$} & $0.21 \pm 0.02$ & 0.1901 \\
\hline$K^{-}$ & {$[42]$} & $0.13 \pm 0.013$ & 0.09981 \\
\hline$K_{S}^{0}$ & {$[42]$} & $0.18 \pm 0.04$ & 0.1382 \\
\hline$\eta$ & & & 0.3918 \\
\hline$\omega$ & & & 0.3514 \\
\hline$\phi$ & [29] & $0.012 \pm 0.0015$ & 0.01593 \\
\hline$\eta^{\prime}$ & & & 0.02576 \\
\hline$\rho^{+}$ & & & 0.4736 \\
\hline$\rho^{-}$ & & & 0.3118 \\
\hline$\rho^{0}$ & & & 0.4254 \\
\hline$K^{*+}$ & & & 0.07360 \\
\hline$K^{*-}$ & & & 0.02976 \\
\hline$K^{* 0}$ & & & 0.06192 \\
\hline $\bar{K}^{* 0}$ & & & 0.03383 \\
\hline$p$ & & & 1.126 \\
\hline $\bar{p}$ & {$[42]$} & $0.040 \pm 0.007$ & 0.04364 \\
\hline$\Delta^{++}$ & & & 0.2937 \\
\hline $\bar{\Delta}^{--}$ & & & 0.007650 \\
\hline$\Lambda$ & [43] & $0.115 \pm 0.012$ & 0.1123 \\
\hline $\bar{\Lambda}$ & {$[43]$} & $0.0148 \pm 0.0019$ & 0.01453 \\
\hline$\Sigma^{+}$ & & & 0.03480 \\
\hline$\Sigma^{-}$ & & & 0.02310 \\
\hline$\Sigma^{0}$ & & & 0.03004 \\
\hline $\bar{\Sigma}^{-}$ & & & 0.003317 \\
\hline $\bar{\Sigma}^{+}$ & & & 0.004384 \\
\hline $\bar{\Sigma}^{0}$ & & & 0.003989 \\
\hline$\Xi^{-}$ & & & 0.001874 \\
\hline$\Xi^{0}$ & & & 0.002119 \\
\hline $\bar{\Xi}^{+}$ & & & 0.0006902 \\
\hline $\bar{\Xi}^{0}$ & & & 0.0006376 \\
\hline$\Omega$ & & & 0.00003783 \\
\hline $\bar{\Omega}$ & & & 0.00002908 \\
\hline$\Lambda(1520)$ & {$[44]$} & $0.012 \pm 0.003$ & 0.009155 \\
\hline$\Theta^{+}$ & & & 0.005224 \\
\hline $\bar{\Theta}^{-}$ & & & 0.0001515 \\
\hline
\end{tabular}




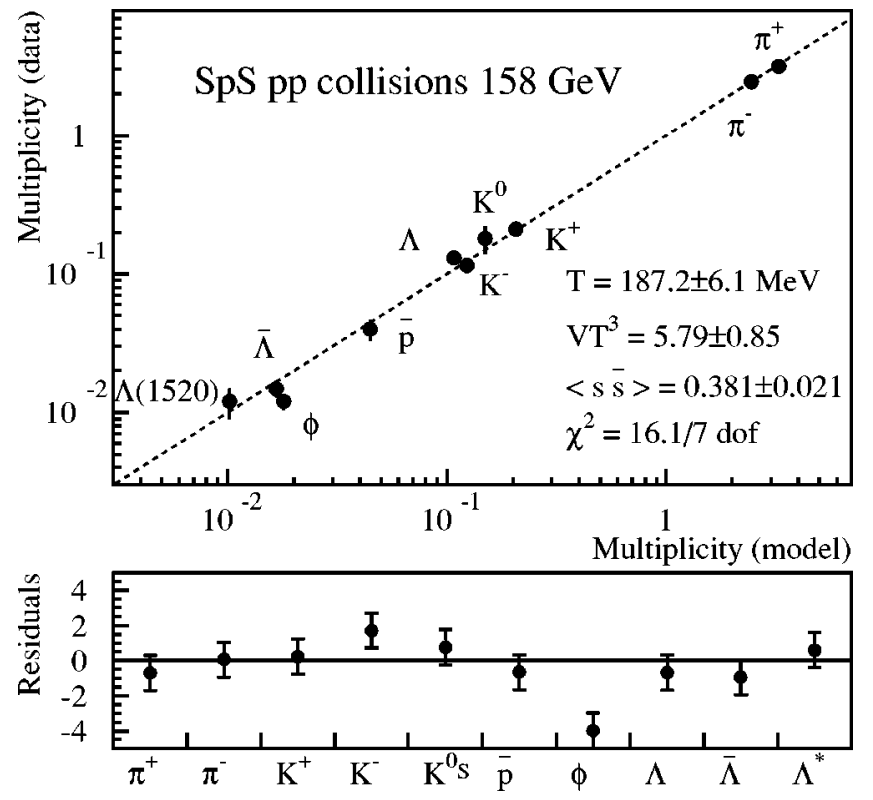

FIG. 9. Above: measured vs fitted multiplicities in the statistical model supplemented with strangeness suppression in $p p$ collisions at a beam energy of $158 \mathrm{GeV}$ corresponding to $\sqrt{s}=17.2 \mathrm{GeV}$; also quoted the best-fit parameters. Below: residual distribution.

$6.45 \mathrm{fm}$. This simple estimate is in approximate agreement with the calculations performed with the Glauber model [35]. Since the number of single-interacting nucleons is expected to be weakly dependent on center-of-mass energy, the fits to this two-component model should yield consistent values of $\left\langle N_{c}\right\rangle$ at $30 \mathrm{~A}, 40 \mathrm{~A}$, and $80 \mathrm{~A} \mathrm{GeV}$ collisions. However, no measurement of hadron production in NN collisions at those energies is available and this question cannot be tackled for the present.

\section{Nonequilibrium of hadrons with light quarks}

An extension of the statistical model has been proposed where QGP hadronization is essentially a statistical coalescence of quarks occurring at an energy density value which does not correspond to a hadron gas at equilibrium [36]. In this model two nonequilibrium parameters are introduced for the different types of quarks, $\gamma_{q}$ for $u, d$ quarks and $\gamma_{s}$ for strange quarks (the difference between $\gamma_{s}$ and $\gamma_{S}$ is explained below). The multiplicity of each hadron thus reads

$$
\begin{aligned}
\left\langle n_{j}\right\rangle= & \frac{\left(2 J_{j}+1\right) V}{(2 \pi)^{3}} \sum_{n=1}^{\infty} \gamma_{s}^{n n_{s}} \gamma_{q}^{n n_{q}} \int d^{3} p \\
& \times \exp \left[-n \sqrt{p^{2}+m_{j}^{2}} / T+n \boldsymbol{\mu} \cdot \mathbf{q}_{j} / T\right],
\end{aligned}
$$

where $n_{s}$ is the number of valence $s$ quarks and $n_{q}$ the number of valence $u, d$ quarks; $\boldsymbol{\mu}$ and $\mathbf{q}_{j}$ are as in Eq. (1). By defining

$$
\gamma_{S}=\frac{\gamma_{s}}{\gamma_{q}}, \quad \tilde{V}=V \gamma_{q}^{2},
$$

the Boltzmann limit of average multiplicity reads

$$
\begin{aligned}
\left\langle n_{j}\right\rangle= & \frac{\left(2 J_{j}+1\right) \tilde{V}}{(2 \pi)^{3}} \gamma_{S}^{n_{s}} \gamma_{q}^{\left|B_{j}\right|} \int d^{3} p \\
& \times \exp \left[-\sqrt{p^{2}+m_{j}^{2}} / T+\boldsymbol{\mu} \cdot \mathbf{q}_{j} / T\right],
\end{aligned}
$$

where $B_{j}$ is the baryon number, as long as mesons have two and baryons have three valence quarks. By comparing this formula with the Boltzmann limit of Eq. (1) it can be realized that the introduction of a light quark nonequilibrium parameter amounts to introducing in the statistical model an overall enhancement (or suppression) of baryons with respect to mesons, unlike in the model $\operatorname{SHM}\left(\gamma_{S}\right)$. We henceforth refer to this model as $\operatorname{SHM}\left(\gamma_{S}, \gamma_{q}\right)$.

The parameter $\gamma_{q}$ has a definite physical bound for bosons which can be obtained by requiring the convergence of the series $\sum_{N=0}^{\infty}\left(\gamma_{q}^{n_{q}{ }^{N}}\right) \exp \left(-N \epsilon / T+N \boldsymbol{\mu} \cdot \mathbf{q}_{j} / T\right)$ for any value of the energy. If the number of $u, d$ quarks to be hadronized is so large that $\gamma_{q}$ is to attain its bounding value, a Bose condensation of particles in the lowest momentum state sets in. For low strangeness and electrical chemical potentials, such as those found in the present analysis, the bounding value is $\gamma_{q}=\exp (m / 2 T)$, where $m$ is the neutral pion mass, e.g., $\gamma_{q}$ $\simeq 1.5$ for $T \simeq 160 \mathrm{MeV}$.

With the introduction of $\gamma_{q}$ as an additional free parameter, there are five parameters to be determined in the model. This makes the minimization procedure rather unstable because it becomes easier to be trapped in local minima. To avoid this, we have performed four parameter fits with fixed values of $\gamma_{q}$ varying from 0.6 to 1.7 in steps of 0.1 . This method allows a clear-cut determination of the absolute minimum.

The results of these fits are shown in Fig. 10 in terms of the minimum $\chi^{2}$ obtained for fixed $\gamma_{q}$. The round dots show the minimum $\chi^{2}$,s achieved by using the main sample of multiplicities in $\mathrm{Pb}-\mathrm{Pb}$ collisions at $158 \mathrm{~A} \mathrm{GeV}$. We find a steady decreasing trend in the value of best-fit temperatures varying from $\simeq 187 \mathrm{MeV}$ at $\gamma_{q}=0.6$ to $\simeq 140 \mathrm{MeV}$ at $\gamma_{q}$ $=1.6$. The fitted temperature at $\gamma_{q}=0.5$ reaches the upper limit of $200 \mathrm{MeV}$, which is the maximum allowed in the model to prevent from being critically dependent on the hadron mass spectrum cutoff. On the other hand, the best-fit values of $\mu_{B} / T$ and $\gamma_{S}$ are rather stable and about the same found in the main fit with $\gamma_{q}=1$. The number of terms in the series (15) has been truncated to five for all particles; the contribution of further terms has been found to be negligible throughout.

It is seen that the absolute $\chi^{2}$ minimum falls in the region of pion condensation, marked by a vertical line at $\gamma_{q} \simeq 1.62$, with $\chi^{2} \simeq 13$ and $T \simeq 140 \mathrm{MeV}$. This finding is in agreement with what is found in Ref. [36]. However, there is also a local minimum at the lower edge $\gamma_{q}=0.6$, with a temperature of $187 \mathrm{MeV}$, which is only one unit of $\chi^{2}$ higher than the absolute minimum. This indicates that the absolute minimum could be rather unstable against variations of the input data and this is in fact what we find by varying down the pion multiplicities by only $1 \sigma$. For this case, the minimum $\chi^{2}$ 's are shown in Fig. 10 as triangular dots and the absolute minimum now lies at $\gamma_{q}=0.6$ instead of at the pion condensation point. 


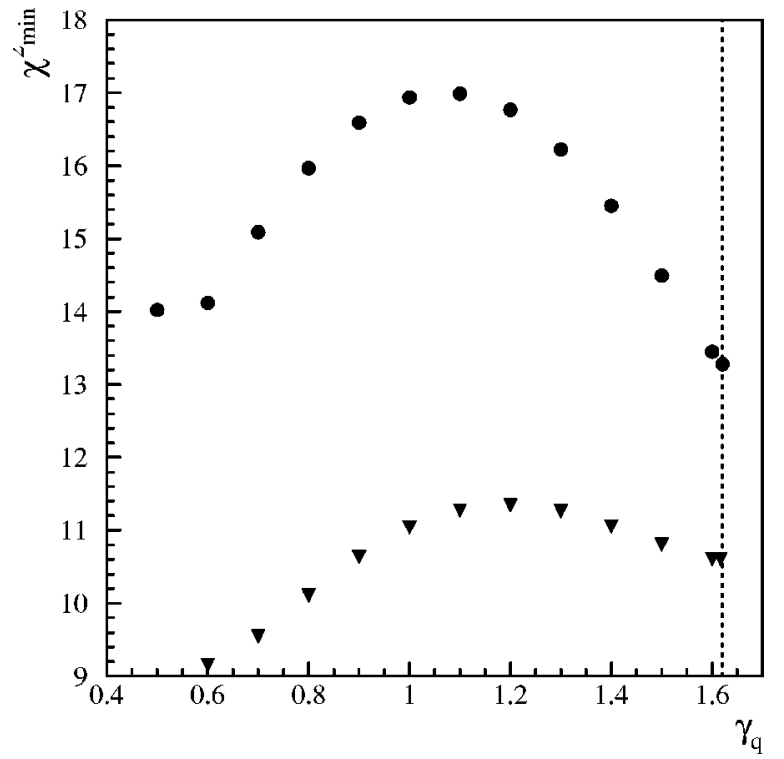

FIG. 10. Minimum $\chi^{2}$ of multiplicity fits in $\mathrm{Pb}-\mathrm{Pb}$ collisions at a beam energy of $158 \mathrm{~A} \mathrm{GeV}$ as a function of a fixed light quark nonequilibrium parameter $\gamma_{q}$. The round dots are the $\chi^{2}$ 's obtained with the main data sample, while triangular dots are those obtained with pion multiplicities lowered by one standard deviation and all others unchanged. The vertical dashed line indicates the condensation point.

In view of the instability of the fit, and of the small relative $\chi^{2}$ improvement in comparison with the main fit, we conclude that there is so far no evidence for the need of this further nonequilibrium parameter. However, it is interesting to note that this model predicts an enhanced (if $\gamma_{q}>1$ ) or suppressed (if $\gamma_{q}<1$ ) production of the $\Theta^{+}$pentaquark baryon with respect to the other versions of the statistical model, even though they agree in reproducing the multiplicities of all other hadrons. This is owing to an additional $\gamma_{q}^{2}$ factor for this special hadron having five valence quarks. From Eqs. (15) and (16) one gets the following, in the Boltzmann limit:

$$
\left\langle n_{\Theta^{+}}\right\rangle=\frac{\gamma_{q}^{3} \gamma_{S} \tilde{V}}{\pi^{2}} m^{2} T \mathrm{~K}_{2}\left(\frac{m}{T}\right) \exp \left[\mu_{B} / T+\mu_{Q} / T+\mu_{S} / T\right]
$$

In fact, as can be seen in Table $\mathrm{V}$ the predicted yield of $\Theta^{+}$ at the global minimum $\gamma_{q} \simeq 1.62$ is more than a factor of two higher than for $\operatorname{SHM}\left(\gamma_{S}\right)$.

As a final remark, we stress that minimum $\chi^{2}$ fits are very useful tools to get information on the state of the source at chemical freeze-out, but, as already emphasized in Sec. III, the simple multiplicity analysis with global parameters resides on an idealization of the collision (e.g., the assumed existence of an EGC) which cannot exactly fit physical reality and discrepancies are to be expected anyway. Thus, a new mechanism or a modification of the basic scheme proves to be relevant only if it leads to a major improvement of the agreement with the data. Slight improvements of the $\chi^{2}$, whenever their significance is beyond its expected statistical

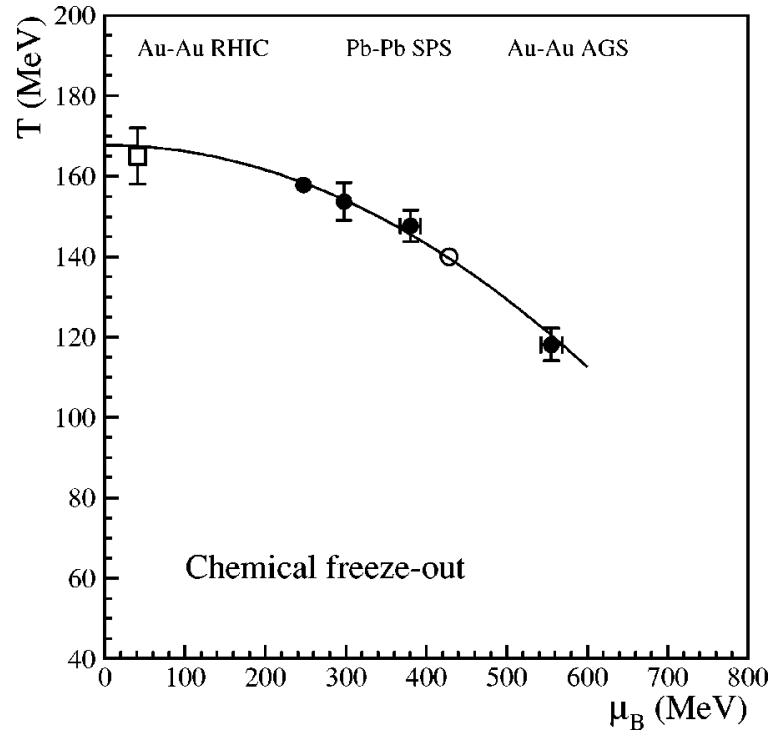

FIG. 11. Chemical freeze-out points in the $\mu_{B}-T$ plane in various heavy ion collisions. The full round dots refer to $\mathrm{Au}-\mathrm{Au}$ at $11.6 \mathrm{~A}$ and $\mathrm{Pb}-\mathrm{Pb}$ collisions at $40 A, 80 A, 158 \mathrm{~A} \mathrm{GeV}$ obtained in the analysis A, while the hollow square dot has been obtained in Ref. [11] by using particle ratios measured at midrapidity in $\mathrm{Au}-\mathrm{Au}$ collisions at ${\sqrt{s_{N N}}}=130 \mathrm{GeV}$. The hollow round dot without error bars refers to $\mathrm{Pb}-\mathrm{Pb}$ collisions at $30 \mathrm{~A} \mathrm{GeV}$ and has been obtained by forcing $T$ and $\mu_{B}$ to lie on the parabola fitted to the full round dots.

fluctuations, cannot be seriously taken as a proof of the validity of a particular scheme.

\section{ENERGY DEPENDENCE}

The statistical model does not make any prediction on the energy dependence of hadron production; its relevant parameters have to be determined separately for each energy and reaction type. Nevertheless, the analysis of the data within this model may help in the study of energy dependence of hadron production because it effectively reduces the full experimental information on numerous hadron yields to only few parameters. Clearly this reduction should be taken with care, were not for the approximate validity of some relevant assumptions, such as the reducibility to EGC (see Sec. II). Furthermore, the reduction procedure may remove or dilute essential physical information. With these caveats in mind, in this section we discuss the energy dependence of the chemical freeze-out parameters extracted from the data (by using the full data sets) within our main $\operatorname{SHM}\left(\gamma_{S}\right)$ approach.

The chemical freeze-out points in the $\mu_{B}-T$ plane are shown in Fig. 11. The RHIC point at ${\sqrt{s_{N N}}}=130 \mathrm{GeV}$, obtained by fitting particle yield ratios at midrapidity, has been taken from Ref. [11]. The four points at beam energies of $11.6 A, 40 A, 80 A$, and $158 A \mathrm{GeV}$ have been fitted with a parabola:

$$
T=0.167-0.153 \mu_{B}^{2},
$$

where $T$ and $\mu_{B}$ are in $\mathrm{GeV}$. The $\mathrm{Pb}-\mathrm{Pb}$ point at $30 A \mathrm{GeV}$ has been forced to lie on the above curve, as has been mentioned in the preceding section. The RHIC point cal- 


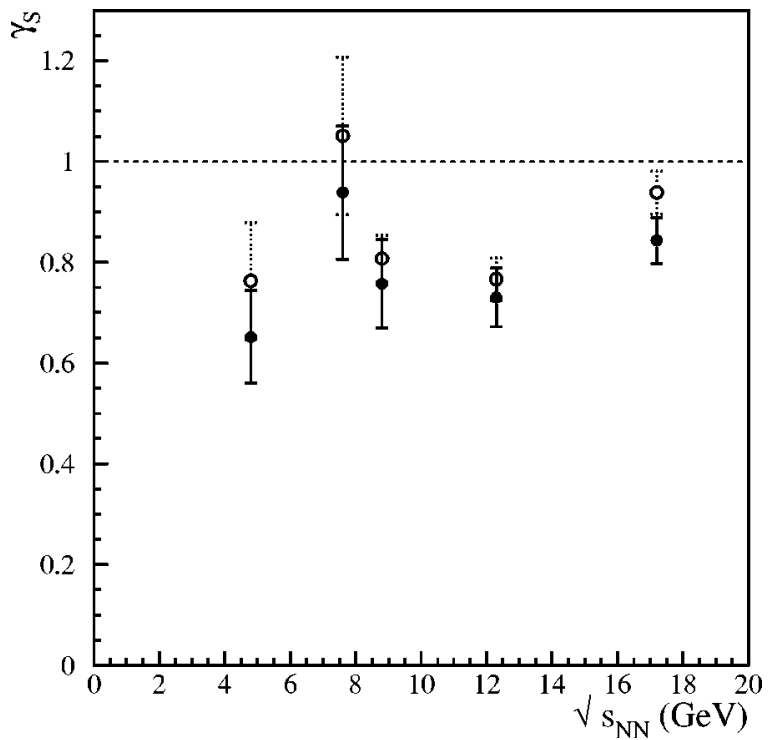

FIG. 12. Strangeness nonequilibrium parameter $\gamma_{S}$ as a function of the nucleon-nucleon center-of-mass energy. Full dots refer to fit A, hollow dots to fit B.

culated in Ref. [11] is in good agreement with the extrapolation of the curve (19).

In the search for deconfinement phase transition, strangeness production is generally believed to be a major item, especially if an anomalous abrupt change was found as a function of center-of-mass energy or other related quantities. A possible indication of it in $\mathrm{Pb}-\mathrm{Pb}$ collisions at the low SPS energies was reported on the basis of the observed energy dependence of several observables [17]. Particularly, the $\left\langle K^{+}\right\rangle /\left\langle\pi^{+}\right\rangle$ratio shows a peaked maximum at about $30 A \mathrm{GeV}$. One may expect that this anomaly should be reflected in the energy dependence of $\gamma_{S}$ parameter fitted within $\operatorname{SHM}\left(\gamma_{S}\right)$ scheme. This dependence is plotted in Fig. 12 and in fact a maximum shows up at $30 \mathrm{~A} \mathrm{GeV}$. Although the error bars are large enough so as to make $\gamma_{S}$ seemingly consistent with a constant as a function of center-of-mass energy, it is important to note that the dominant systematic errors on experimental data at SPS energies are essentially common. Therefore, the errors on the model parameters at different SPS energies turn out to be strongly correlated, hence fitted $\gamma_{S}$ 's are expected to move up or down together.

In order to further study strangeness production features, we have also compared the measured $\left\langle K^{+}\right\rangle /\left\langle\pi^{+}\right\rangle$ratio (including the preliminary RHIC result at $\sqrt{s}_{N N}=200 \mathrm{GeV}$ [37]) with the theoretical values in a hadron gas along the freezeout curve (19) as a function of the fitted baryon-chemical potential for different values of $\gamma_{S}$ (see Fig. 13). The calculated dependence of $\left\langle K^{+}\right\rangle /\left\langle\pi^{+}\right\rangle$on $\mu_{B}$ is nonmonotonic with a broad maximum at $\mu_{B} \simeq 400 \mathrm{MeV}$ (i.e., $E_{\text {beam }} \simeq 30 \mathrm{~A} \mathrm{GeV}$ ) [38]. Taking into account that systematic errors at different energies in $\mathrm{Pb}-\mathrm{Pb}$ collisions are fully correlated, we can conclude that the data points seem not to follow the constant $\gamma_{S}$ lines.

In fact, the anomalous increase of relative strangeness production at $30 \mathrm{~A} \mathrm{GeV}$ can be seen also in the Wroblewski variable $\lambda_{S}=2\langle s \bar{s}\rangle /(\langle u \bar{u}\rangle+\langle d \bar{d}\rangle)$, the estimated ratio of newly

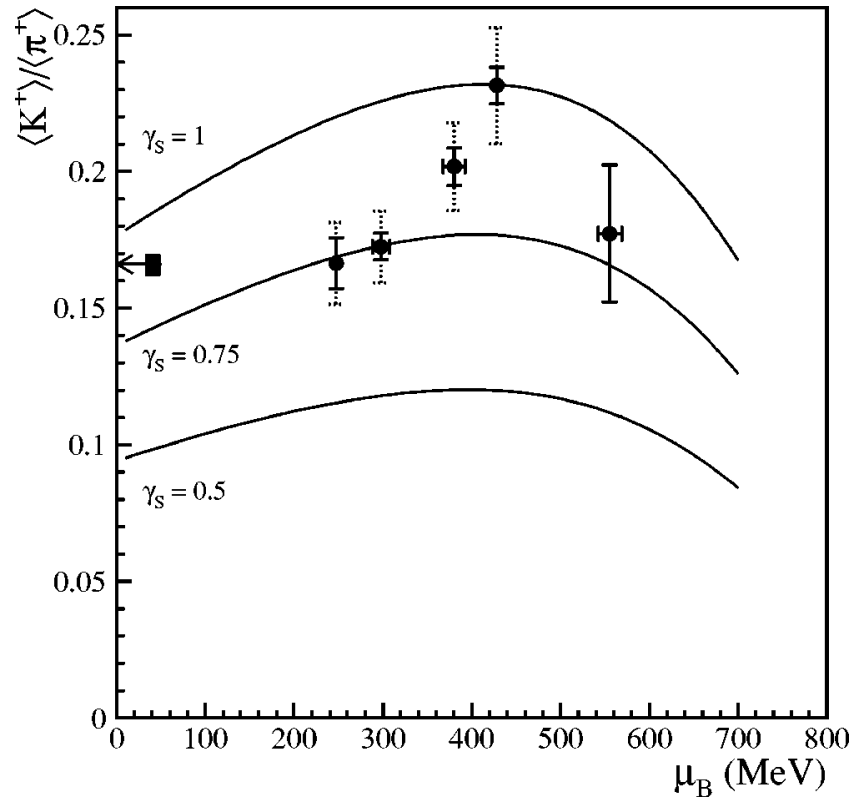

FIG. 13. Measured $\left\langle K^{+}\right\rangle /\left\langle\pi^{+}\right\rangle$ratio as a function of the fitted baryon-chemical potential. The full square dot is a preliminary full phase space measurement in $\mathrm{Au}-\mathrm{Au}$ collisions at $\sqrt{s}_{N N}=200 \mathrm{GeV}$ [37] and the error is only statistical; the arrow on the left signifies that its associated baryon chemical potential is lower than that estimated at $\sqrt{s}_{N N}=130 \mathrm{GeV}$ [11] used here. For the SPS energy points the statistical errors are indicated with solid lines, while the contribution of the common systematic error is shown as a dotted line. Also shown are the theoretical values for a hadron gas along the fitted chemical freeze-out curve shown in Fig. 11, for different values of $\gamma_{S}$.

produced strange quarks to $u, d$ quarks at primary hadron level, shown in Fig. 14 and Table VII. The calculation of newly produced quark pairs is performed by using the statistical model best-fit values of the various hadron multiplicities, so the obtained $\lambda_{S}$ values are somehow model dependent. Nevertheless, this variable features a very similar behavior as the ratio $\left\langle K^{+}\right\rangle /\left\langle\pi^{+}\right\rangle$and attains a maximum value of 0.61 at $30 \mathrm{~A} \mathrm{GeV}$, very close to that predicted for $\gamma_{S}=1$.

These deviations from a smooth behavior of strangeness production are certainly intriguing, yet the analysis within the SHM will be more conclusive in this regard with a larger data sample at $30 \mathrm{~A} \mathrm{GeV}$ and at the forthcoming $20 \mathrm{~A} \mathrm{GeV}$ data.

\section{SUMMARY AND CONCLUSIONS}

We presented a detailed study of chemical freeze-out conditions in ultrarelativistic heavy ion collisions at projectile momenta of $11.6 A$ (Au-Au at AGS), 30A, 40A, 80A, and $158 A(\mathrm{~Pb}-\mathrm{Pb}$ at $\mathrm{SPS}) \mathrm{GeV}$, corresponding to nucleon-nucleon center-of-mass energies of 4.8, 7.6, 8.8, 12.3, $17.2 \mathrm{GeV}$, respectively. By analyzing hadronic multiplicities measured in full phase space within the statistical hadronization model, we have tested and compared different versions of this model, with special emphasis on possible explanations of the observed strangeness under-saturation at the hadron level. 


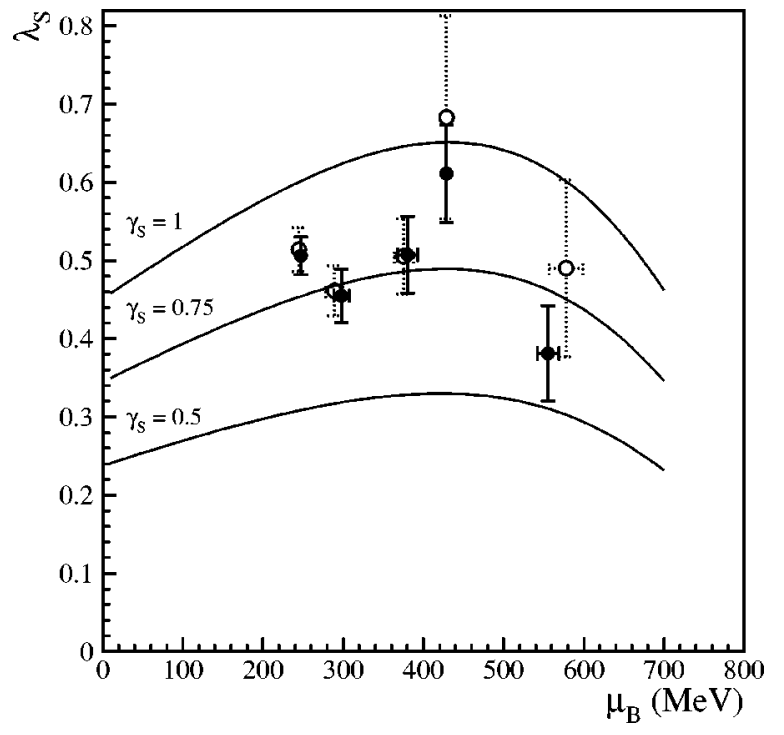

FIG. 14. $\lambda_{S}$ estimated from the fits A (full dots) and B (hollow dots) as a function of the fitted baryon-chemical potential. Also shown are the theoretical values for a hadron gas along the fitted chemical freeze-out curve shown in Fig. 11, for different values of $\gamma_{S}$.

It is found that version of the model referred to as SHM $\left(\gamma_{S}\right)$, where a nonequilibrium population of hadron carrying strange valence quarks is allowed, fits all the data analyzed in this paper. We have also shown that the seeming full chemical equilibrium found for central $\mathrm{Pb}+\mathrm{Pb}$ collisions at $158 \mathrm{~A} \mathrm{GeV}$ by using particle yields integrated over a limited region around midrapidity is most likely an artefact of the kinematical cut.

We have tested a model [SHM(TC)] in which hadron production is pictured as stemming from two independent components: a fireball (or a set of fireballs) at full chemical equilibrium and single nucleon-nucleon interactions. This model can fit the data at $158 \mathrm{~A} \mathrm{GeV}$ if the number of collisions is around 50 with a sizeable uncertainty. So far, it cannot be confirmed at other energies due to the lack of the precise data on NN interactions.

A model in which strangeness is assumed to vanish locally [34] yields a worse fit to the data with respect to SHM $\left(\gamma_{S}\right)$ and $\operatorname{SHM}(\mathrm{TC})$.

Finally, we have also tested a model in which a nonequilibrium population of hadrons carrying both strange and light valence quarks is allowed. We have found that the present set of available data does not allow us to establish whether a further nonequilibrium parameter is indeed needed to account for the observed hadron production pattern. A discriminating prediction of this model with respect to the $\operatorname{SHM}\left(\gamma_{S}\right)$ is an enhanced production of the recently discovered $\Theta^{+}$pentaquark baryon due to the additional factor $\gamma_{q}^{2}$.

Energy dependence of chemical freeze-out parameters has been discussed based on the results obtained with our main version of the model $\operatorname{SHM}\left(\gamma_{S}\right)$. The evolution of the freezeout temperature and baryon-chemical potential is found to be smooth in the AGS-SPS-RHIC energy range. The strangeness-suppression parameter $\gamma_{S}$ is found to be smaller than one $\left(\gamma_{S} \simeq 0.8\right)$ for most of the studied collisions which confirms previous findings [6,9,27], with an indication of a maximum at $30 \mathrm{~A} \mathrm{GeV}$, where $\gamma_{S}$ is found to be close to one. The significance of this maximum is related to the correlation between errors on hadron yield measurements at different energies. The interpolated dependence of relative strangeness production on energy and baryon-chemical potential, as measured by the $\left\langle K^{+}\right\rangle /\left\langle\pi^{+}\right\rangle$ratio and the Wroblewski factor $\lambda_{S}$, features a broad maximum at about $30 \mathrm{~A}$ of beam energy. However, the experimental measurement of $\left\langle K^{+}\right\rangle /\left\langle\pi^{+}\right\rangle$and the estimated $\lambda_{S}$ value in $\mathrm{Pb}-\mathrm{Pb}$ collisions at this energy seemingly exceed the expected values for a fixed $\gamma_{S}$.

\section{ACKNOWLEDGMENTS}

We would like to thank Jens Ivar Jørdre for his help with $\left\langle K^{+}\right\rangle /\left\langle\pi^{+}\right\rangle$ratio at RHIC. This work was part of INFN research Project No. FI31.

\section{APPENDIX A: FROM CANONICAL TO GRAND-CANONICAL ENSEMBLE}

The canonical partition function of the $i$ th cluster can be written as a multiple integral over the interval $[\pi, \pi][13]$,

$$
Z_{i}\left(\mathbf{Q}_{i}\right)=\frac{1}{(2 \pi)^{3}} \int d^{3} \phi e^{\mathrm{i} \mathbf{Q}_{i} \cdot \phi} \exp [F(\phi)],
$$

where $\mathbf{Q}_{i}=\left(Q_{i}, B_{i}, S_{i}\right)$ is a vector having as components the electric charge, baryon number, and strangeness of the cluster, $\phi=\left(\phi_{Q}, \phi_{B}, \phi_{S}\right)$, and $F(\phi)$ reads

$$
F(\phi)=\sum_{j} \frac{\left(2 J_{j}+1\right) V_{i}}{(2 \pi)^{3}} \int d^{3} p \ln \left(1 \pm e^{-\sqrt{p^{2}+m_{j}^{2}} / T-\mathrm{i} \phi \cdot \mathbf{q}_{j}}\right)^{ \pm 1},
$$

where $V_{i}$ is the volume and $T$ the temperature of the cluster; the sum runs over all hadronic species $j$ and $\mathbf{q}_{j}$ the charge vector of the $j$ th hadron; the upper sign applies to fermions, the lower to bosons. The probability distribution required for the reduction to EGC to apply reads $[6,13]$

$$
w\left(\mathbf{Q}_{1}, \ldots, \mathbf{Q}_{N}\right)=\frac{\prod_{i} Z_{i}\left(\mathbf{Q}_{i}\right) \delta_{\Sigma_{i} \mathbf{Q}_{i}, \mathbf{Q}}}{\sum_{\mathbf{Q}_{1}, \ldots, \mathbf{Q}_{N}} \prod_{i} Z_{i}\left(\mathbf{Q}_{i}\right) \delta_{\Sigma_{i} \mathbf{Q}_{i}, \mathbf{Q}}}
$$

In this case, the overall multiplicity of the $j$ th hadron is given by [13]

$$
\left\langle n_{j}\right\rangle=\left.\frac{\partial}{\partial \lambda_{j}} \ln Z(\mathbf{Q})\right|_{\lambda_{j}=1},
$$

where $Z(\mathbf{Q})$ is the canonical partition function of the equivalent global cluster:

$$
Z(\mathbf{Q})=\sum_{\mathbf{Q}_{1}, \ldots, \mathbf{Q}_{N}} \prod_{i} Z_{i}\left(\mathbf{Q}_{i}\right) \delta_{\Sigma_{i} \mathbf{Q}_{i}, \mathbf{Q}}
$$

and $\lambda_{j}$ is a fictitious fugacity parameter. Formally, this turns out to be the same function as in Eq. (A1) with $V=\Sigma_{i} V_{i}$ replacing $V_{i}$ and $\mathbf{Q}=\Sigma_{i} \mathbf{Q}_{i}$ replacing $\mathbf{Q}_{i}$. If $V$ is large, the 
canonical partition function can be approximated by the leading term of an asymptotic saddle-point expansion. Setting $\exp \left(-\mathrm{i} \phi_{k}\right)=z_{k}$, the canonical partition function can be written as

$$
Z(\mathbf{Q})=\frac{1}{(2 \pi i)^{3}}\left[\prod_{k=1}^{3} \oint \frac{d z_{k}}{z_{k}}\right] \exp \left[F_{c}(\mathbf{z})\right] \prod_{k=1}^{3} z_{k}^{-Q_{k}},
$$

where

$$
F_{c}(\mathbf{z})=\sum_{j} \frac{\left(2 J_{j}+1\right) V_{i}}{(2 \pi)^{3}} \int d^{3} p \ln \left(1 \pm e^{-\sqrt{p^{2}+m_{j}^{2}} / T} \prod_{k=1}^{3} z_{k}^{q_{j k}}\right)^{ \pm 1} .
$$

The saddle-point expansion is carried out by requiring the logarithmic derivative of the integrand to vanish:

$$
-\frac{Q_{k}}{z_{k}}+\frac{\partial F_{c}}{\partial z_{k}}=0, \quad k=1,2,3 .
$$

The solutions of this equation are indeed the grand-canonical fugacities $\lambda_{k} \equiv \exp \left(\mu_{k} / T\right)$. The function $F_{c}(\boldsymbol{\lambda})$ coincides with the logarithm of the grand-canonical partition function $\ln Z_{g c}$, therefore Eq. (A8) expresses the equality between the average charge in the grand-canonical ensemble $\lambda_{k} \partial \log Z_{g c} / \partial \lambda_{k}$ and the initial value $Q_{k}$. The canonical partition function now becomes, at the second order of the expansion,

$$
\begin{aligned}
Z(\mathbf{Q}) \simeq & \exp \left[F_{c}(\boldsymbol{\lambda})\right]\left[\prod_{k=1}^{3} \lambda_{k}^{-Q_{k}}\right] \frac{1}{(2 \pi \mathrm{i})^{3}}\left[\prod_{k=1}^{3} \oint \frac{d z_{k}}{z_{k}}\right] \\
& \times \exp \left[-(\mathbf{z}-\boldsymbol{\lambda})^{T} \mathrm{H}(\mathbf{z}-\boldsymbol{\lambda}) / 2\right],
\end{aligned}
$$

where $\mathrm{H}$ is the Hessian matrix in $z_{k}=\lambda_{k}$. The first exponential factor is just the grand-canonical partition function $Z_{g c}$ calculated for the fugacities $\lambda_{k}$. The average multiplicity of the $j$ th hadron species can now be calculated by using Eq. (A4) taking the approximated expression (A9) of the canonical partition function. Retaining only the dominant contribution, one just obtains the grand-canonical expression of the average multiplicity as expressed in Eq. (1).

APPENDIX B: PROOF OF EQ. (10)

The argument closely follows the previous one. The main difference is the request of vanishing strangeness for each cluster. Thus, the configurational probabilities, Eq. (A3), w turn to

$$
w\left(\mathbf{Q}_{1}, \ldots, \mathbf{Q}_{N}\right)=\frac{\prod_{i} Z_{i}\left(\mathbf{Q}_{i}\right) \delta_{\Sigma_{i} \mathbf{Q}_{i}, \mathbf{Q}} \delta_{S_{i}, 0}}{\sum_{\mathbf{Q}_{1}, \ldots, \mathbf{Q}_{N}} \prod_{i} Z_{i}\left(\mathbf{Q}_{i}\right) \delta_{\Sigma_{i} \mathbf{Q}_{i}, \mathbf{Q}} \delta_{S_{i}, 0}}
$$

and the average multiplicity of the $j$ th hadron species now reads

$$
\left\langle n_{j}\right\rangle=\left.\frac{\partial}{\partial \lambda_{j}} \ln \sum_{\mathbf{Q}_{1}, \ldots, \mathbf{Q}_{N}} \prod_{i} Z\left(\mathbf{Q}_{i}\right) \delta_{\mathbf{Q}, \Sigma_{i} \mathbf{Q}_{i}} \delta_{S_{i}, 0}\right|_{\lambda_{j}=1},
$$

where $\lambda_{j}$ is a fictitious fugacity. Let us now work out the expression

$$
\zeta=\sum_{\mathbf{Q}_{1}, \ldots, \mathbf{Q}_{N}} \prod_{i=1}^{N} Z_{i}\left(\mathbf{Q}_{i}\right) \delta_{\mathbf{Q}, \Sigma_{i} \mathbf{Q}_{i}} \delta_{S_{i}, 0}
$$

by assuming that all the clusters have the same volume $V_{c}$ and temperature $T$. This can be done rewriting the canonical partition functions $Z_{i}$ like in Eq. (A1), using the integral representation of Kronecker's $\delta$ and eliminating the redundant strangeness conservation constraints. Thus, expanding the vector $\mathbf{Q}$ in its components, Eq. (B3) becomes

$$
\begin{aligned}
\zeta= & \sum_{B_{1}, Q_{1}, \ldots, B_{N}, Q_{N}} \int_{-\pi}^{\pi} \frac{d \phi_{B}}{2 \pi} \int_{-\pi}^{\pi} \frac{d \phi_{Q}}{2 \pi} e^{i B \phi_{B}+i Q \phi_{Q}} \\
& \times \exp \left(-i \sum_{i} B_{i} \phi_{B}-i \sum_{i} Q_{i} \phi_{Q}\right) \\
& \times \prod_{i=1}^{N} \int_{-\pi}^{\pi} \frac{d \phi_{i B}}{2 \pi} \int_{-\pi}^{\pi} \frac{d \phi_{i Q}}{2 \pi} \int_{-\pi}^{\pi} \frac{d \phi_{i S}}{2 \pi} \\
& \times e^{i B_{i} \phi_{i B}+i Q_{i} \phi_{i Q} \exp \left[F\left(\phi_{i B}, \phi_{i Q}, \phi_{i S}\right)\right],}
\end{aligned}
$$

where $F$ is the function in Eq. (A2) with $V_{c}$ replacing $V_{i}$. Note that this function is the same for all clusters, $T$ and $V_{c}$ being constant. We can now carry out the sum over all the integers $B_{i}, Q_{i}$ in Eq. (B4) and get

$$
\begin{gathered}
\sum_{B_{1}, Q_{1}, \ldots, B_{N}, Q_{N}} \exp \left(-i \sum_{i} B_{i}\left(\phi_{B}-\phi_{i B}\right)-i \sum_{i} Q_{i}\left(\phi_{Q}-\phi_{i Q}\right)\right) \\
=\prod_{i=1}^{N}(2 \pi)^{3} \delta\left(\phi_{B}-\phi_{i B}\right) \delta\left(\phi_{Q}-\phi_{i Q}\right)
\end{gathered}
$$

so that the integration over $\phi_{i B}$ and $\phi_{i Q}$ in Eq. (B4) can be easily done and one is left with

$$
\begin{aligned}
\zeta= & \int_{-\pi}^{\pi} \frac{d \phi_{B}}{2 \pi} \int_{-\pi}^{\pi} \frac{d \phi_{Q}}{2 \pi} e^{i B \phi_{B}+i Q \phi_{Q}} \\
& \times \prod_{i=1}^{N} \int_{-\pi}^{\pi} \frac{d \phi_{i S}}{2 \pi} \exp \left[F\left(\phi_{B}, \phi_{Q}, \phi_{i S}\right)\right] .
\end{aligned}
$$

As the function $F$ is the same for all clusters, this can be written also as

$$
\begin{aligned}
\zeta= & \int_{-\pi}^{\pi} \frac{d \phi_{B}}{2 \pi} \int_{-\pi}^{\pi} \frac{d \phi_{Q}}{2 \pi} e^{i B \phi_{B}+i Q \phi_{Q}} \\
& \times\left\{\int_{-\pi}^{\pi} \frac{d \phi_{S}}{2 \pi} \exp \left[F\left(\phi_{B}, \phi_{Q}, \phi_{S}\right)\right]\right\}^{V / V_{c}},
\end{aligned}
$$

being $V=\Sigma_{i} V_{i}=N V_{c}$. For large volumes, we can approximate $\zeta$ by means of the saddle-point expansion of the integrals over $\phi_{B}$ and $\phi_{Q}$ like in Appendix A. Thus, similarly to Eq. (A9), 


$$
\begin{aligned}
\zeta \propto & \lambda_{B}^{-B} \lambda_{Q}^{-Q}\left\{\int _ { - \pi } ^ { \pi } \frac { d \phi _ { S } } { 2 \pi } \operatorname { e x p } \left[\sum_{j} \frac{\left(2 J_{j}+1\right) V_{c}}{(2 \pi)^{3}}\right.\right. \\
& \left.\times \int d^{3} p \ln \left(1 \pm \lambda_{B}^{B_{j}} \lambda_{Q}^{Q_{j}} e^{\left.-\sqrt{p^{2}+m_{j}^{2}} / T-i \phi_{S} S_{j}\right)^{ \pm 1}}\right]\right\}^{V / V_{c}} .
\end{aligned}
$$

The function

$$
\begin{aligned}
& Z_{c} \equiv \int_{-\pi}^{\pi} \frac{d \phi_{S}}{2 \pi} \exp \left[\sum_{j} \frac{\left(2 J_{j}+1\right) V_{c}}{(2 \pi) 3}\right. \\
& \times \int d^{3} p \ln \left(1 \pm \lambda_{B}^{B_{j}} \lambda_{Q}^{Q_{j}} e^{\left.-\sqrt{p^{2}+m_{j}^{2}} / T-i \phi_{S} S_{j}\right)^{ \pm 1}}\right]
\end{aligned}
$$

is defined as the strange canonical partition function. The multiplicity of the hadron $j$ can now be calculated by means of Eq. (B2) by using Eqs. (B3) and (B7) and the definition (B9). What is obtained is just Eq. (10).
[1] S. V. Afanasiev et al., NA49 Collaboration, Phys. Rev. C 66, 054902 (2002).

[2] C. Alt et al., NA49 Collaboration, nucl-ex/0305017.

[3] M. Gazdzicki and M. I. Gorenstein, Acta Phys. Pol. B 30, 2705 (1999).

[4] J. Cleymans and H. Satz, Z. Phys. C 57, 135 (1993).

[5] J. Sollfrank, M. Gaździcki, U. Heinz, and J. Rafelski, Z. Phys. C 61, 659 (1994).

[6] F. Becattini, M. Gaździcki, and J. Sollfrank, Eur. Phys. J. C 5, 143 (1998)

[7] P. Braun-Munzinger, I. Heppe, and J. Stachel, Phys. Lett. B 465, 15 (1999).

[8] G. D. Yen and M. I. Gorenstein, Phys. Rev. C 59, 2788 (1999).

[9] F. Becattini, J. Cleymans, A. Keränen, E. Suhonen, and K. Redlich, Phys. Rev. C 64, 024901 (2001).

[10] P. Braun-Munzinger et al., Phys. Lett. B 518, 41 (2001).

[11] W. Florkowski, W. Broniowski, and M. Michalec, Acta Phys. Pol. B 33, 761 (2002).

[12] F. Becattini, Z. Phys. C 69, 485 (1996).

[13] F. Becattini and U. Heinz, Z. Phys. C 76, 269 (1997).

[14] F. Becattini and G. Passaleva, Eur. Phys. J. C 23, 551 (2002).

[15] R. Hagedorn, Nucl. Phys. B24, 93 (1979); R. Stock, Phys. Lett. B 456, 277 (1999).

[16] M. van Leeuwen et al., NA49 Collaboration, Nucl. Phys. A715, 161c (2003).

[17] V. Friese et al., NA49 Collaboration, nucl-ex/0305017; M. Gazdzicki, hep-ph/0305176.

[18] R. Hagedorn, lectures delivered at CERN on the Thermodynamics of Strong Interactions, 1970 (unpublished); cern-th/ $7190 / 94$

[19] A. Keränen and F. Becattini, Phys. Rev. C 65, 044901 (2002).

[20] P. Koch, B. Müller, and J. Rafelski, Phys. Rep. 142, 167 (1986).

[21] K. Hagiwara et al., Phys. Rev. D 66, 010001 (2002).

[22] S. V. Afanasiev et al., Nucl. Phys. A715, 161 (2003).

[23] S. Albergo et al., Phys. Rev. Lett. 88, 062301 (2002).

[24] S. Ahmad et al., Phys. Lett. B 382, 35 (1996).

[25] L. Ahle et al., E802 Collaboration, Phys. Rev. C 60, 064901
(1999).

[26] B. B. Back et al., E917 Collaboration, Phys. Rev. Lett. 87, 242301 (2001)

[27] J. Cleymans, B. Kaempfer, and S. Wheaton, Phys. Rev. C 65, 027901 (2002).

[28] S. V. Afanasiev et al., Nucl. Phys. A715, 453 (2003).

[29] S. V. Afanasev et al., NA49 Collaboration, Phys. Lett. B 491, 59 (2000).

[30] S. V. Afanasiev et al., NA49 Collaboration, Phys. Lett. B 538, 275 (2002).

[31] F. Antinori et al., WA97 Collaboration, Nucl. Phys. A661, 130c (1999); Numerical values in http://wa97.web.cern.ch/ WA97/Data/TableQM99.html

[32] F. Becattini, J. Phys. G 28, 1553 (2002).

[33] H. Appelshäuser et al., NA49 Collaboration, Eur. Phys. J. C 2, 661 (1998).

[34] S. Hamieh, K. Redlich, and A. Tounsi, Phys. Lett. B 486, 61 (2000).

[35] C. Höhne (private communication).

[36] J. Letessier and J. Rafelski, Phys. Rev. C 59, 947 (1999).

[37] D. Ouerdane, Ph. D. thesis, NBI Copenhagen, Denmark, 2003 (unpublished); J. I. Jørdre, BRAHMS Collaboration, in Proceedings of the EPS2003 Conference, Aachen, Germany, 2003 (unpublished).

[38] P. Braun-Munzinger, J. Cleymans, H. Oeschler, and K. Redlich, Nucl. Phys. A697, 902 (2002).

[39] L. Ahle et al., E802 Collaboration, Phys. Rev. C 60, 044904 (1999).

[40] L. Ahle et al., E802 Collaboration, Phys. Rev. C 59, 2173 (1999).

[41] V. Friese, NA49 Collaboration, Nucl. Phys. A698, 487 (2002).

[42] J. Bachler et al., NA49 Collaboration, Nucl. Phys. A661, 45 (1999).

[43] A. Billmeier, NA49 Collaboration, Ph. D. thesis, University of Frankfurt, Germany, 2001 (unpublished).

[44] S. V. Afanasev et al., NA49 Collaboration, J. Phys. G 27, 367 (2001). 